Check for updates

Cite this: Phys. Chem. Chem. Phys., 2018, 20, 24577

Received 20th June 2018, Accepted 20th August 2018

DOI: $10.1039 / c 8 c p 03915 a$

rsc.li/pccp

\section{Narrowing the gap between experimental and computational determination of methyl group dynamics in proteins $\dagger$}

\author{
Falk Hoffmann, (D) a Mengjun Xue, (D) ${ }^{b}$ Lars V. Schäfer (D) *a and \\ Frans A. A. Mulder (iD *b
}

\begin{abstract}
Nuclear magnetic resonance (NMR) spin relaxation has become the mainstay technique to sample protein dynamics at atomic resolution, expanding its repertoire from backbone ${ }^{15} \mathrm{~N}$ to side-chain ${ }^{2} \mathrm{H}$ probes. At the same time, molecular dynamics (MD) simulations have become increasingly powerful to study protein dynamics due to steady improvements of physical models, algorithms, and computational power. Good agreement between generalized Lipari-Szabo order parameters derived from experiment and MD simulation has been observed for the backbone dynamics of a number of proteins. However, the agreement for the more dynamic side-chains, as probed by methyl group relaxation, was much worse. Here, we use T4 lysozyme (T4L), a protein with moderate tumbling anisotropy, to showcase a number of improvements that reduce this gap by a combined evaluation of NMR relaxation experiments and MD simulations. By applying a protein force field with accurate methyl group rotation barriers in combination with a solvation model that yields correct protein rotational diffusion times, we find that properly accounting for anisotropic protein tumbling is an important factor to improve the match between NMR and MD in terms of methyl axis order parameters, spectral densities, and relaxation rates. The best agreement with the experimentally measured relaxation rates is obtained by a posteriori fitting the appropriate internal time correlation functions, truncated by anisotropic overall tumbling. In addition, MD simulations led us to account for a hitherto unrealized artifact in deuterium relaxation experiments arising from strong coupling for leucine residues in uniformly ${ }^{13} \mathrm{C}$-enriched proteins. For $\mathrm{T} 4 \mathrm{~L}$, the improved analysis reduced the RMSD between MD and NMR derived methyl axis order parameters from 0.19 to 0.11 . At the level of the spectral density functions, the improvements allow us to extract the most accurate parameters that describe protein side-chain dynamics. Further improvement is challenging not only due to force field and sampling limitations in MD, but also due to inherent limitations of the Lipari-Szabo model to capture complex dynamics.
\end{abstract}

\section{Introduction}

The biological function of proteins is often intimately connected with their dynamical behavior. ${ }^{1,2}$ NMR spectroscopy is a powerful technique to characterize protein motions on different time scales, both site-specifically and globally, ${ }^{3-7}$ and combining NMR spectroscopy with molecular dynamics (MD) simulations is particularly useful, as MD simulations can help to interpret NMR relaxation data. ${ }^{8-10}$ In this paper, several advances in the

\footnotetext{
${ }^{a}$ Theoretical Chemistry, Ruhr-University Bochum, D-44780 Bochum, Germany. E-mail: lars.schaefer@ruhr-uni-bochum.de; Tel: +492343221582

${ }^{b}$ Interdisciplinary Nanoscience Center (iNANO) and Department of Chemistry, University of Aarhus, DK-8000 Aarhus, Denmark. E-mail: fmulder@chem.au.dk; Tel: +4587155889

$\dagger$ Electronic supplementary information (ESI) available. See DOI: 10.1039/c8cp03915a
}

analysis of protein dynamics from methyl deuterium spin relaxation and MD simulations will be described, which results in an improved agreement between experiments and computations.

The motion of a bond vector is described by its time correlation function (TCF)

$$
C(t)=\left\langle P_{2}[\vec{\mu}(0) \cdot \vec{\mu}(t)]\right\rangle
$$

where $P_{2}(x)=\left(3 x^{2}-1\right) / 2$ is the second order Legendre polynomial, $\vec{\mu}(t)$ is the unit vector of the bond, and $\langle\cdots\rangle$ denotes the average over all time step differences $t$. The total TCF describes the reorientation motion of the bond in the laboratory frame. In addition to the internal dynamics, a bond also moves together with the full protein. As a consequence, bond orientations become uncorrelated on time scales longer than the overall rotational diffusion (tumbling) correlation time $\tau_{\mathrm{R}}$ of the protein, and the TCF decays to 0 for $t \gg \tau_{\mathrm{R}}$. The square of the generalized 
order parameter $S^{2}$ describes the internal dynamics of the bond in the molecular frame, and is defined as the long-time limit of the internal TCF,

$$
S^{2}=\lim _{t \rightarrow \infty} C_{\text {int }}(t)
$$

hence disregarding overall tumbling of the molecule. $S^{2}$ is a measure of the amplitude of internal reorientation motions and varies between 0 and 1 , with lower values indicating largeamplitude motions of a mostly unrestricted bond vector and higher values corresponding to restricted motions of a bond that is (mostly) rigid in the molecular frame.

In an NMR spin relaxation experiment, relaxation rates are measured. These relaxation rates determine the spectral densities $J(\omega)$ at discrete frequencies that depend on the Larmor frequencies of the involved nuclear spins, as the stochastic internal and overall motions provide the energy for transitions. Fourier transformation of the TCF yields the power spectral density,

$$
J(\omega)=\int_{0}^{\infty} C(t) \mathrm{e}^{-i \omega t} \mathrm{~d} t
$$

thus connecting relaxation and molecular dynamics. Since the spectral density is only sampled at a few specific frequencies in NMR relaxation, it is not possible to experimentally determine the complete TCF. Furthermore, because the internal dynamics are convoluted with overall tumbling, one cannot measure $C_{\text {int }}(t)$ separately. The order parameter for the long-time limit, as defined by eqn (2), is thus not directly accessible in NMR relaxation experiments in solution.

Lipari and Szabo (LS) ${ }^{11,12}$ described simplified analytical models for interpreting NMR relaxation experiments in terms of generalized order parameters and associated correlation times. The LS formalism, which is related to work by Halle and Wennerström, ${ }^{13}$ assumes that internal motions and overall tumbling are statistically independent, which is for example the case if they occur on sufficiently different time scales. In this case, the total TCF can be factorized into the internal and overall TCFs, $C(t)=C_{\text {int }}(t) C_{\mathrm{O}}(t)$. For a molecule that tumbles isotropically in solution with a global rotational tumbling time $\tau_{\mathrm{R}}$, the overall motion can be described by a single-exponential decay, $C_{\mathrm{O}}(t)=\mathrm{e}^{-t / \tau_{\mathrm{R}}}$. Lipari and Szabo assumed that the internal TCF can also be described by a single-exponential decay on a characteristic time scale of fast internal dynamics, $\tau_{\mathrm{f}} \ll$ $\tau_{\mathrm{R}}$. Using the reduced time $\tau_{\text {red }}=\left(\tau_{\mathrm{R}} \tau_{\mathrm{f}}\right) /\left(\tau_{\mathrm{R}}+\tau_{\mathrm{f}}\right)$, the internal and total TCFs in the original LS model are approximated by

$$
C_{\text {int }}(t)=S^{2}+\left(1-S^{2}\right) \mathrm{e}^{-t / \tau_{\mathrm{f}}}
$$

and

$$
C(t)=S^{2} \mathrm{e}^{-t / \tau_{\mathrm{R}}}+\left(1-S^{2}\right) \mathrm{e}^{-t / \tau_{\text {red }}}
$$

respectively. The corresponding LS spectral density $J(\omega)$ is a sum of Lorentzians,

$$
J(\omega)=\frac{2}{5}\left(\frac{S^{2} \tau_{\mathrm{R}}}{1+\left(\omega \tau_{\mathrm{R}}\right)^{2}}+\frac{\left(1-S^{2}\right) \tau_{\mathrm{red}}}{1+\left(\omega \tau_{\mathrm{red}}\right)^{2}}\right) .
$$

This model describes the motion of a bond with a single fast internal motion time scale. Later, Clore and coworkers ${ }^{14}$ extended this model to internal motions on two time scales $\tau_{\mathrm{f}} \ll \tau_{\mathrm{s}}$, which are separated by at least one order of magnitude. In this case, the internal and total time correlation functions are

$$
C_{\text {int }}(t)=S^{2}+\left(1-S_{\mathrm{f}}^{2}\right) \mathrm{e}^{-t / \tau_{\mathrm{f}}}+\left(S_{\mathrm{f}}^{2}-S^{2}\right) \mathrm{e}^{-t / \tau_{\mathrm{s}}}
$$

and

$$
C(t)=S^{2} \mathrm{e}^{-t / \tau_{\mathrm{R}}}+\left(1-S_{\mathrm{f}}^{2}\right) \mathrm{e}^{-t / \tau_{\mathrm{f}, \mathrm{red}}}+\left(S_{\mathrm{f}}^{2}-S^{2}\right) \mathrm{e}^{-t / \tau_{\mathrm{s}, \mathrm{red}}}
$$

with the reduced times $\tau_{\mathrm{f}, \text { red }}=\left(\tau_{\mathrm{R}} \tau_{\mathrm{f}}\right) /\left(\tau_{\mathrm{R}}+\tau_{\mathrm{f}}\right)$ and $\tau_{\mathrm{s}, \text { red }}=\left(\tau_{\mathrm{R}} \tau_{\mathrm{s}}\right) /$ $\left(\tau_{\mathrm{R}}+\tau_{\mathrm{s}}\right)$ for fast and slow internal motions, respectively. Assuming that the fast internal motions are axially symmetric and independent of the slow motions, the full order parameter can be factorized into the order parameters for fast and slow internal motions, $S^{2}=S_{\mathrm{f}}{ }^{2} S_{\mathrm{s}}{ }^{2} \cdot{ }^{14}$ If $\tau_{\mathrm{f}}$ is sufficiently short, the second term in eqn (8) can be neglected and the spectral density in the extended LS model is

$$
J(\omega)=\frac{2}{5}\left(\frac{S^{2} \tau_{\mathrm{R}}}{1+\left(\omega \tau_{\mathrm{R}}\right)^{2}}+\frac{\left(S_{\mathrm{f}}^{2}-S^{2}\right) \tau_{\mathrm{s}, \mathrm{red}}}{1+\left(\omega \tau_{\mathrm{s}, \mathrm{red}}\right)^{2}}\right) .
$$

The spectral density function is used to fit the spectral density points determined by the measured relaxation rates at discrete Larmor frequencies. In the isotropic model, $\tau_{\mathrm{R}}$ is assumed to be a single global tumbling time, whereas in case of moderate anisotropy, residue-specific $\tau_{\mathrm{R}}$ 's may be used (see below). The LS2 model involves two fitting parameters, $S^{2}$ and $\tau_{\mathrm{f}}$. If the internal motions occur on a comparable time scale as the overall tumbling (or even slower), the two types of motions are inseparable. In the three-parameter LS3 model, $\tau_{\mathrm{c}}^{\text {eff }}$ is thus used as an additional fitting parameter instead of a fixed $\tau_{\mathrm{R}}$ in LS2. The TCF and spectral density function in the LS3 model are

$$
C(t)=S^{2} \mathrm{e}^{-t / \tau_{\mathrm{c}}^{\text {eff }}}+\left(1-S^{2}\right) \mathrm{e}^{-t / \tau_{\text {red }}}
$$

and

$$
J(\omega)=\frac{2}{5}\left(\frac{S^{2} \tau_{\mathrm{c}}^{\mathrm{eff}}}{1+\left(\omega \tau_{\mathrm{c}}^{\mathrm{eff}}\right)^{2}}+\frac{\left(1-S^{2}\right) \tau_{\mathrm{red}}}{1+\left(\omega \tau_{\mathrm{red}}\right)^{2}}\right)
$$

respectively, with $\tau_{\text {red }}=\left(\tau_{\mathrm{c}}^{\text {eff }} \tau_{\mathrm{f}}\right) /\left(\tau_{\mathrm{c}}^{\text {eff }}+\tau_{\mathrm{f}}\right)$. The extended LS model with four fitting parameters $\left(S_{\mathrm{f}}{ }^{2}, \tau_{\mathrm{f}}, S_{\mathrm{s}}{ }^{2}, \tau_{\mathrm{s}}\right)$ easily leads to overfitting in the analysis of side-chain relaxation data ${ }^{15}$ and was therefore not used in our analysis.

The present work is concerned with the dynamics of methyl groups in protein side-chains. To probe methyl dynamics by deuterium spin relaxation experiments in $\mathrm{NMR},{ }^{16,17}$ one measures the relaxation rates of fractionally deuterated methyl groups in protein side-chains, which are sensitive to the reorientation motions of ${ }^{13} \mathrm{C}-{ }^{2} \mathrm{H}$ bond vectors. For example, the longitudinal, in-phase transverse magnetization, and quadrupolar order rates,

$$
R\left(D_{z}\right)=\frac{3}{16}\left(\frac{q Q e^{2}}{\hbar}\right)^{2}\left[J\left(\omega_{\mathrm{D}}\right)+4 J\left(2 \omega_{\mathrm{D}}\right)\right]
$$

$$
R\left(D_{y}\right)=\frac{1}{32}\left(\frac{q Q e^{2}}{\hbar}\right)^{2}\left[9 J(0)+15 J\left(\omega_{\mathrm{D}}\right)+6 J\left(2 \omega_{\mathrm{D}}\right)\right]
$$


and

$$
R\left(3 D_{z}^{2}-2\right)=\frac{3}{16}\left(\frac{q Q e^{2}}{\hbar}\right)^{2}\left[3 J\left(\omega_{\mathrm{D}}\right)\right]
$$

respectively, are converted into spectral densities at multiples of the Larmor frequency of deuterium $\omega_{\mathrm{D}}$. Hence, fitting these points to the spectral density function as defined by eqn (6) or eqn (11) yields generalized order parameters and associated correlation times. Deuterium relaxation is dominated by interactions between the nuclear quadrupole moment $e Q$ and the electric field gradient $e q$, as reflected in the quadrupolar coupling constant $\left(q Q e^{2} / \hbar\right)^{2}$ in the above equations.

The dynamics of methyl groups comprises the motions of the $\mathrm{C}_{\text {methyl }}{ }^{2} \mathrm{H}_{\text {methyl }}$ bonds in addition to the dynamics of the $\mathrm{C}-\mathrm{C}_{\text {methyl }}$ (or $\mathrm{S}-\mathrm{C}_{\text {methyl }}$, for methionine) bonds that connect the methyl group to the rest of the side-chain. The bonds can undergo different kinds of motions, which are reflected in the corresponding TCFs. Methyl motions include (1) very fast $\mathrm{C}_{\text {methyl }}{ }^{2} \mathrm{H}_{\text {methyl }}$ librations on the sub-ps time scale; these motions usually decrease the time correlation function to a value of about 0.9 within less than 1 ps. ${ }^{18}$ (2) Rapid jumps around the 3 -fold methyl symmetry axis, typically within less than 100 ps. For ideal tetrahedral methyl geometry this decreases the TCF of the $\mathrm{C}-{ }^{2} \mathrm{H}$ bond to a value of $1 / 9 C(t=0)$. (3) Angular librational motions of the entire side-chain and fast rotamer jumps on the sub-ns time scale, which leads to a further decay of the $\mathrm{C}_{\text {methyl }}{ }^{2} \mathrm{H}_{\text {methyl }}$ internal TCF to its limiting value of $1 / 9 S_{\text {axis }}{ }^{2}$ in the absence of slow internal motions (see point 4); here, $S_{\text {axis }}{ }^{2}$ is the methyl order parameter of the symmetry axis. (4) Slow internal dynamics of the entire sidechain due to jumps between different rotamer states on a broad range of time scales up to several ns and beyond. Methyl order parameters lower than 0.7 indicate population of more than a single rotamer well. ${ }^{19-22}$ Internal motions that occur on time scales longer than $\tau_{\mathrm{R}}$ do not affect ${ }^{2} \mathrm{H}$ NMR quadrupolar relaxation, because the TCF has already decayed to zero due to global tumbling. Nevertheless, it is these motions that determine the decay of the internal TCF to its final value according to eqn (2). (5) Overall tumbling of the protein. While isotropic tumbling can be described by a single-exponential decay with a global rotational tumbling time $\tau_{\mathrm{R}}$, in case of moderate anisotropy one may consider residue-specific $\tau_{\mathrm{R}}$ 's (see below).

MD simulations are routinely used to calculate backbone order parameters $S_{\mathrm{NH}}{ }^{2}$ with high accuracy. However, methyl group order parameters are more challenging and less frequently reported in the literature. Kasinath and coworkers ${ }^{23}$ used an analogous approach to the long-time limit of $C_{\text {int }}(t)$ of the $\mathrm{C}-\mathrm{C}_{\text {methyl }}$ bond, eqn (2). They investigated seven proteins (comprising of 73 to 168 residues) in MD simulations of lengths between 112 ns and 260 ns with the CHARMM $27^{24}$ force field. The reported average Pearson correlation coefficient for methyl order parameters (with respect to experiment) is 0.65 for the seven investigated proteins. Bowman ${ }^{25}$ simulated ubiquitin and compared three force fields (AMBER ff03, ${ }^{26}$ AMBER ff99SB-ILDN, ${ }^{27}$ and $\mathrm{CHARMM} 27^{24}$ ) and three methods (long-time limit approach according to eqn (2), a related truncated-average approximation that employs the average of the internal TCF close to the molecular tumbling time $\tau_{\mathrm{R}}$, and the Lipari-Szabo model of the internal TCF, eqn (4)). He concluded that microseconds of simulation time are required to obtain statistically reliable methyl order parameters and, furthermore, that the agreement to NMR between the MD-derived methyl order parameters obtained with the longtime limit approach decreases with increasing simulation time, whereas the opposite is the case for the other approaches. Prompers and coworkers ${ }^{28}$ proposed the isotropic reorientational eigenmode dynamics (iRED) model, in which a principal component analysis of covariance matrices of backbone $\mathrm{N}-\mathrm{H}$ vectors and their corresponding 2nd rank spherical harmonics from MD trajectories yield reorientational eigenmodes of the protein. The different eigenmodes correspond to correlated dynamics of the bond vectors, and the eigenvalue spectrum shows whether the time scales of internal and overall motions are separable. Genheden $^{29}$ tested iRED for methyl groups and reported mean unsigned deviations of methyl axis order parameters from experiment of 0.13 and 0.17 for galectin-3 in complex with lactose or a synthetic derivate, respectively.

Most of the proteins studied previously may be described as fairly isotropic. These proteins have the advantage that the tumbling time of all individual methyl groups is close to the global rotational tumbling time $\tau_{\mathrm{R}}$ of the protein. Accounting for anisotropy is more challenging. ${ }^{30-37}$ Lipari and Szabo already mentioned in their original work ${ }^{12}$ that anisotropy should be accounted for if the macromolecule has a non-spherical shape. In that case, overall tumbling cannot be captured by an exponential decay with a single global time constant $\tau_{\mathrm{R}}$, but needs to be described by an anisotropic diffusion tensor. Importantly, the resulting generalized order parameters are defined with respect to the principal axis frame (PAF) of the protein diffusion tensor. To assess rotational diffusion anisotropy in proteins, Ryabov and coworkers ${ }^{38}$ analyzed a set of 841 protein structures from the Protein Data Bank (PDB), chosen as a representative set of globular single-domain folds. They found that only $11 \%$ of 753 prolate diffusion tensors had small anisotropies $1<A<$ 1.17 and could be approximated as isotropic for the purposes of NMR studies. Here, $A=D_{\|} / D_{\perp}$ is the ratio of the diffusion constants along parallel and perpendicular directions of the diffusion tensor. $68 \%$ of the tensors had intermediate anisotropies in the range $1.17<A<1.6$, indicating that, in general, it is imperative to take anisotropic diffusion into account for accurate analysis of NMR relaxation data. As these typical anisotropies are in the applicability range of the quadratic approximation of Brüschweiler and Wright, ${ }^{39}$ residue-specific correlation times can be calculated based on the overall diffusion tensor and knowledge of the 3D structure. The fitting of diffusion tensors in the analysis of ${ }^{15} \mathrm{~N}$ relaxation data is commonplace since several decades, ${ }^{40,41}$ but is often overlooked in the analysis of ${ }^{2} \mathrm{H}$ relaxation data. However, this approach can also be utilized for side-chain vectors, provided that the direction of the methyl symmetry axis can be accurately determined.

Only a few studies have considered taking anisotropic tumbling into account. Millet and coworkers ${ }^{42}$ compared the 
difference in the experimentally derived order parameters for the $\mathrm{C}-\mathrm{C}_{\text {methyl }}$ bonds of protein $\mathrm{L}$ using an isotropic approach with a single global rotational tumbling time $\tau_{\mathrm{R}}$ for all methyl groups, and an anisotropic approach with residue-specific $\tau_{\mathrm{R}, i}$ values for every methyl group. They found that the effect of anisotropy was small, but can lead to order parameter differences of 0.05 for ALA residues. Skrynnikov and coworkers ${ }^{15}$ found a qualitatively similar result. Ishima and coworkers ${ }^{43}$ have shown that for HIV-1 protease, a protein with intermediate anisotropy $\left(D_{\|} / D_{\perp}=1.35\right)$, the use of the isotropic model led to errors up to $12 \%$ in $S_{\text {axis }}{ }^{2}$. Yet, the effect of anisotropic overall tumbling when analyzing side-chain dynamics is not considered in many studies of methyl relaxation.

Wong and coworkers ${ }^{33}$ proposed a method to evaluate protein rotational diffusion from MD simulations. The rotational tumbling time $\tau_{\mathrm{R}}$ cannot just be approximated by $1 /\left(6 D_{\text {iso }}\right)$ with the isotropic rotational diffusion constant $D_{\text {iso. In general, }}$ diffusion is described by a rank-2 tensor $\mathscr{D}$ (i.e., a $3 \times 3$ matrix). Diagonalization yields the principal components of $\mathscr{D}$ in its principal axis frame (PAF); the trace of this tensor is $3 D_{\text {iso. }}$ For a protein that can be approximated as an axially symmetric rotor (i.e., prolate or oblate), the difference from this isotropic value is characterized by the principal components for diffusion of the long $\left(D_{\|}\right)$and short $\left(D_{\perp}\right)$ axes.

A realistic description of the overall rotational diffusion tensor in an MD simulation critically depends on the water model, which has a significant effect on the tumbling times. For example, Maragakis and coworkers ${ }^{44}$ calculated backbone $\mathrm{NH}$ order parameters from a $1.2 \mu \mathrm{s}$ simulation of ubiquitin using the OPLS-AA force field and the SPC water model by fitting to the Lipari-Szabo TCF (eqn (5)). They reported a rotational tumbling time of $\tau_{\mathrm{R}}=1.98 \mathrm{~ns}$, which is a factor of 2 smaller than the experimental tumbling time. This is in line with the faster self-diffusion of SPC water in comparison to real water. The overall tumbling does not affect the calculation of $S^{2}$ from eqn (2), but can affect the order parameters obtained from eqn (6). This is especially the case for bond motions that occur on time scales that are comparable to the overall tumbling correlation time of the protein, see below.

In the present work, we use NMR relaxation and MD simulations to derive methyl order parameters $S_{\text {axis }}{ }^{2}$ as well as relaxation rates for $\mathrm{T} 4$ lysozyme $(\mathrm{T} 4 \mathrm{~L})$, a prolate protein with intermediate anisotropy $\left(D_{\|} / D_{\perp} \approx 1.4\right)$. We compare different approaches to obtain $S_{\text {axis }}{ }^{2}$ from MD simulations. First, we use the long-time limit approach of eqn (2), which has been widely used for methyl order parameters in the literature. ${ }^{22,23,45-50}$ Second, we use the Lipari-Szabo TCF fitting approach (eqn (5) for LS2, eqn (10) for LS3), which was used for backbone order parameters recently, ${ }^{25,44}$ but to our knowledge has not been extended to methyls. Third, we compare these methods with a spectral density mapping approach that is similar in nature to the one used to analyze the experimental NMR relaxation data. The generalized order parameters obtained from the MD simulations are compared to those obtained from ${ }^{2} \mathrm{H}$ NMR relaxation data. We show that the discrepancy between NMR and $\mathrm{MD}$ can be alleviated by taking the decay of the time correlation functions (and hence spectral densities) due to anisotropic protein tumbling properly into account in the analysis of the MD data. Furthermore, the spectral density approach enables one to extract not only $S_{\text {axis }}{ }^{2}$ from the MD simulations, but - maybe even more importantly - also relaxation rates that can be compared to the ones directly measured by NMR relaxation experiments, without the need to invoke simplified motional models.

In the following, we describe each of the improvements in detail. First, we show that different spectral densities and order parameter values are obtained in the presence of LEU strong coupling. We identify the origin of this artifact, and describe when to remove faulty data. Second, we discuss how anisotropy affects the interpretation of experimental NMR relaxation data in terms of generalized order parameters of methyl groups. We will illustrate with our experimental data that the use of an isotropic model leads to spurious differences in the order parameters of prochiral methyl groups (e.g., VAL-C $\mathrm{C}^{\gamma, 1} / \mathrm{C}^{\gamma, 2}$ or LEU-C $\left.\mathrm{C}^{\delta, 1} / \mathrm{C}^{\delta, 2}\right)$, while an anisotropic model removes these differences. Third, we turn to MD simulations and quantitatively assess the time correlation functions obtained with the Amber ff99SB*-ILDN force field in combination with different water models; in this part we also discuss how anisotropic protein tumbling can be accounted for in the analysis of MD simulations. Then, we compare different ways to calculate generalized methyl order parameters from MD simulations and evaluate their agreement with experimental values. Finally, we show the agreement between the relaxation rates determined by NMR relaxation experiments and MD simulations.

\section{Results and discussion}

\subsection{LEU strong coupling}

In an initial analysis of T4L deuterium relaxation data, very different order parameter values were obtained for the prochiral methyl groups of LEU residues (Table S1, ESI $\dagger$ ). One can see, that the difference in methyl order parameter between the two prochiral LEU methyl groups can be rather large, e.g., the difference between the methyl order parameter $\Delta S_{\text {axis }}{ }^{2}$ of the two prochiral methyl groups ${ }^{13} \mathrm{C}^{\delta, 1}$ and ${ }^{13} \mathrm{C}^{\delta, 2}$ for LEU79 and LEU118 are 0.23 and 0.28, respectively, which seemingly suggests very different internal dynamics of these methyls. We observed that these differences contrasted the MD simulations, which predicted very similar order parameters for prochiral methyl groups. Using the long-time limit approach (see Methods), a method which is insensitive to protein tumbling, we obtained MD methyl order parameter differences $\Delta S_{\text {axis }}{ }^{2}$ between the two prochiral methyl groups for LEU79 and LEU118 of 0.01 and 0.02 , respectively.

We therefore hypothesized that the experimental data were flawed, and strong ${ }^{13} \mathrm{C}-{ }^{13} \mathrm{C}$ coupling in LEU residues might be responsible. Strong coupling can be expected particularly in the case where the LEU side-chain is rigid. In that case, LEU are typically found in one of the two most favorable side-chain conformations mt or $\mathrm{tp}^{51,52}$ (Table S2, ESI $\dagger$ ). This can be recognized from the chemical shift difference of the two prochiral $\mathrm{C}^{\delta}$ methyls 
due to the gamma gauche effect, which reports on the $\chi_{2}$ side-chain angle. ${ }^{53}$ This leads to the methyl group trans to $\mathrm{C}^{\alpha}$ being shifted down field relative to the methyl group in the gauche position. Therefore, in the case of limited LEU side-chain mobility, one of the methyl groups will have a chemical shift in very close proximity to the ${ }^{13} \mathrm{C}^{\gamma}$ chemical shift.

The impact of artifacts arising from strong ${ }^{13} \mathrm{C}-{ }^{13} \mathrm{C}$ coupling on methyl group order parameters can best be demonstrated with the data of chicken $\alpha$-spectrin SH3 from Agarwal ${ }^{54}$ (Table S3, ESI $\dagger$ ). The two methyl order parameter of 6 of the 7 LEU side-chains have been measured for samples produced from $3^{-13} \mathrm{C}$-pyruvate and uniformly ${ }^{13} \mathrm{C}$-labeled glucose. In the sample produced from ${ }^{13} \mathrm{C}_{6}$-glucose LEU methyl groups are ${ }^{13} \mathrm{C}$-enriched, as well as the attached carbons in the side-chain, which is avoided by use of $3{ }^{-13} \mathrm{C}$-pyruvate ${ }^{55}$ such that strong ${ }^{13} \mathrm{C}-{ }^{13} \mathrm{C}$ spin coupling cannot occur. The ${ }^{13} \mathrm{C}^{\delta}$ chemical shift of one of the two methyl groups for all of these side-chains was higher than $24 \mathrm{ppm}$, indicating that these methyl groups could experience strong coupling. In 4 of these 6 cases, the methyl order parameter difference between glucose- and pyruvate-derived samples is larger than 0.1 , while it is always lower than 0.1 for the LEU methyl groups with ${ }^{13} \mathrm{C}^{\delta}$ chemical shifts lower than $24 \mathrm{ppm}$. For example, for LEU8, $S_{\text {axis }}{ }^{2}$ order parameters for the two methyl groups are 0.67 and 0.97 when derived for a uniformly ${ }^{13} \mathrm{C}$-labeled sample. When producing the protein from $3{ }^{13} \mathrm{C}$-pyruvate, these values are 0.61 and 0.71 , respectively, clearly showing the impact of the strong coupling artifact. In the absence of complete chemical shift assignments for the sidechain ${ }^{13} \mathrm{C}$ shifts, therefore, signals appearing within approximately 2-3 standard deviations from the ${ }^{13} \mathrm{C}^{\gamma}$ shift (26 $\pm 1.1 \mathrm{ppm}$ in BMRB) should be eliminated or treated with great caution. Thus, in practice, ${ }^{2} \mathrm{H}$ relaxation data for LEU with ${ }^{13} \mathrm{C}^{\delta}$ chemical shifts larger than 24 ppm may be compromised. Although this was mentioned in a footnote by Xue ${ }^{56}$ an exploration of the effect, to our knowledge, has hitherto not been published.

We measured ${ }^{13} \mathrm{C}$ chemical shifts of all methyl groups of T4L and identified 9 LEU methyl groups with ${ }^{13} \mathrm{C}^{\delta}$ chemical shifts that are close to their respective ${ }^{13} \mathrm{C}^{\gamma}$ chemical shifts. All LEU methyl groups that could be affected by LEU strong coupling (highlighted in red in Table S1, ESI $\dagger$ ) were eliminated from further analysis.

\subsection{NMR relaxation data show anisotropy of T4L}

The three experimental quadrupolar relaxation rates $R\left(D_{z}\right)$, $R\left(D_{y}\right)$, and $R\left(3 D_{z}^{2}-2\right)$ at $950 \mathrm{MHz}$ were measured for the Cys-free wild-type of T4 lysozyme and analyzed with the spectral density mapping approach, where the relaxation rates are converted into spectral density values at the frequencies $0, \omega_{\mathrm{D}}$ and $2 \omega_{\mathrm{D}}$. The LS2 and LS3 models were used to fit the spectral density points. The results for the measured relaxation rates and the fitted model parameters can be found in Table S4 and Fig. S1, ESI. $\dagger$

Table 1 compares the methyl order parameter of specific methyl groups using an isotropic Lipari-Szabo model with a global rotational tumbling time $\tau_{\mathrm{R}}=10.70 \mathrm{~ns}$, and an anisotropic model with methyl group-specific rotational tumbling times. The chosen residues fit well to the LS2 model in both cases.
Table 1 NMR rotational tumbling times $\tau_{\mathrm{R} \text {,iso }}$ and $\tau_{\mathrm{R} \text {,aniso }}$ and generalized methyl order parameters $S_{\text {iso }}{ }^{2}$ and $S_{\text {aniso }}{ }^{2}$ of selected methyl groups with the isotropic (left) and axially symmetric (right) model, respectively. The chosen methyl groups fit well to the LS2 model

\begin{tabular}{|c|c|c|c|c|}
\hline Methyl group & $\tau_{\mathrm{R}, \text { iso }}[\mathrm{ns}]$ & $S_{\text {iso }}{ }^{2}$ & $\tau_{\mathrm{R}, \text { aniso }}[\mathrm{ns}]$ & $S_{\text {aniso }}{ }^{2}$ \\
\hline LEU39-C $^{\delta, 1}$ & 10.70 & 0.63 & 11.00 & 0.62 \\
\hline LEU39-C ${ }^{\delta, 2}$ & 10.70 & 0.54 & 10.14 & 0.57 \\
\hline ALA97-C ${ }^{\beta}$ & 10.70 & 0.79 & 9.69 & 0.88 \\
\hline ALA98-C ${ }^{\beta}$ & 10.70 & 0.94 & 11.57 & 0.88 \\
\hline ILE100-C ${ }^{\gamma, 2}$ & 10.70 & 0.97 & 11.89 & 0.89 \\
\hline ILE100-C $\mathrm{C}^{\delta, 1}$ & 10.70 & 0.63 & 9.77 & 0.68 \\
\hline VAL149-C ${ }^{\gamma, 1}$ & 10.70 & 0.76 & 9.82 & 0.83 \\
\hline VAL149-C ${ }^{\gamma, 2}$ & 10.70 & 0.90 & 11.62 & 0.84 \\
\hline
\end{tabular}

Table 1 shows that the order parameters of the prochiral methyl groups of LEU39 and of VAL149, respectively, are very different in the isotropic model $\left(\Delta S_{\text {axis }}{ }^{2}=0.09\right.$ and 0.14 for LEU39-C $\mathrm{C}^{\delta, 1 / \delta, 2}$ and VAL149-C $\mathrm{C}^{\gamma, 1 / \gamma, 2}$, respectively). Likewise, the order parameters of ALA97-C $\mathrm{C}^{\beta}$ and ALA98-C $\mathrm{C}^{\beta}$, both of which are part of the same $\alpha$-helix, differ by 0.15 in the isotropic model. If one employs an anisotropic model (prolate), derived from backbone ${ }^{15} \mathrm{~N}$ relaxation data, and takes the orientation of the methyl symmetry axis into account, the artificial differences between these order parameters are diminished. This indicates that the methyl groups in the two consecutive ALA residues in the same $\alpha$-helix as well as the prochiral methyl groups in the same side-chain (LEU39, VAL149) have similar internal dynamics, but tumble differently in the reference frame due to their different orientations with respect to the PAF of the protein diffusion tensor. For example, the $\mathrm{C}^{\alpha}-\mathrm{C}^{\beta}$ vector of ALA98 is oriented along the direction of the slowly tumbling long axis of the protein, while the same bond in ALA97 points almost perpendicular to it. Furthermore, artifactually high order parameters such as for ILE100-C $\mathrm{C}^{\gamma, 2}$ can also result from neglecting anisotropic protein tumbling (Table 1).

A proper interpretation of order parameters from NMR relaxation data for proteins of even moderate anisotropy clearly seems to require adequate consideration of overall tumbling.

\subsection{Effect of the water model on protein dynamics in MD simulations}

In principle, MD simulations can provide the spectral density at all frequencies. In practice, this requires accurate time correlation functions, which describe the internal motions and the overall tumbling of the bond in the laboratory frame. Here, we compare two commonly used water models, TIP3P ${ }^{57}$ and TIP4P $/ 2005,{ }^{58}$ in terms of the internal backbone dynamics and the overall tumbling of the protein. For all simulations, the Amber ff99SB*-ILDN force field ${ }^{27,59,60}$ was used for the protein, including recent adjustments of the methyl rotation barriers. ${ }^{61}$ In each case, ten MD simulations were carried out; the simulations with TIP4P/2005 water were 300 ns each, and the simulations with TIP3P were $100 \mathrm{~ns}$ each. Thus, the accumulated simulation times are $3 \mu$ s and $1 \mu$ s for TIP4P/2005 and TIP3P water, respectively, and each individual MD trajectory exceeds $\tau_{\mathrm{R}}$ by about a factor 30 .

Since the Amber force fields were originally parametrized together with the TIP3P model, we first tested the influence of the water model on the backbone order parameters and rotational 


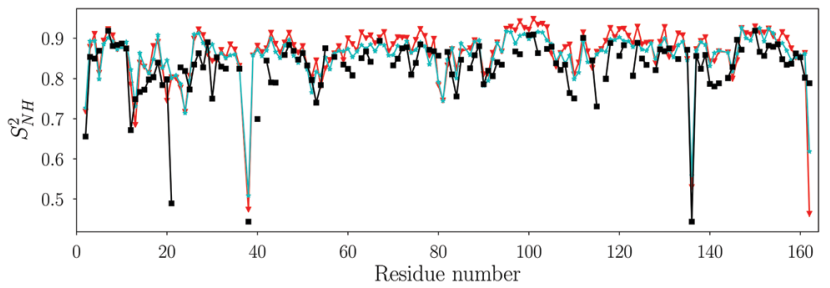

Fig. 1 Order parameter $\mathrm{S}_{\mathrm{NH}}{ }^{2}$ of backbone $\mathrm{N}-\mathrm{H}$ vectors fitted with the Lipari-Szabo TCF model (eqn (10)) for the MD simulations with the TIP3P (cyan stars) and the TIP4P/2005 (red triangles) water models. The Pearson and Spearman correlation coefficients between the TIP3P and TIP4P/2005 $S_{\mathrm{NH}}{ }^{2}$ are $R_{\mathrm{P}}=0.95$ and $R_{\mathrm{S}}=0.89$, respectively. The order parameters from NMR relaxation experiments are shown as black squares. The RMSD between NMR and MD (TIP4P/2005 water) is 0.05 .

tumbling times. We calculated the TCFs of all backbone $\mathrm{N}-\mathrm{H}$ bonds via eqn (1) and extracted $S_{\mathrm{NH}}{ }^{2}$ and corresponding $\tau_{\mathrm{R}, i}$ values by fitting to eqn (10). The rotational tumbling times from eqn (10), which are defined in the laboratory frame, were used together with the initial structure of the MD simulations to calculate the diffusion tensor of the protein using the program QUADRIC (see Methods). Fig. 1 shows the backbone $\mathrm{NH}$ order parameters, and the rotational tumbling times are shown in Fig. S2, ESI. $\dagger$

Fig. 1 shows that the backbone order parameters extracted from the two water models are very similar, and both agree with the experimental order parameters for most $\mathrm{N}-\mathrm{H}$ vectors (see also Table S5, ESI $\dagger$ ). The order parameters from MD are in general a little bit higher than those from NMR. The difference in $S_{\mathrm{NH}}{ }^{2}$ between both water models is less than 0.05 for all non-terminal residues except for ASP40, where it is 0.09. These results show that the water model does not influence the internal dynamics of the backbone, in line with previous findings. ${ }^{29,33,62,63}$

The $\tau_{\mathrm{R}, i}$ values of individual backbone $\mathrm{N}-\mathrm{H}$ vectors are highly correlated between the two water models, but the time scales differ substantially (Fig. S2, ESI $\dagger$ ). The residue-averaged tumbling times are $4.3 \pm 0.3 \mathrm{~ns}$ and $10.9 \pm 0.5 \mathrm{~ns}$ for TIP3P and TIP4P/ 2005 , respectively. This difference by a factor of $c a$. 2.5 coincides with the ratio of the self-diffusion constants of the two water models, which are $6.05 \times 10^{-9} \mathrm{~m}^{2} \mathrm{~s}^{-1}$ and $2.49 \times 10^{-9} \mathrm{~m}^{2} \mathrm{~s}^{-1}$ for TIP3P and TIP4P/2005, respectively. ${ }^{64,65}$ Although the overall rotational tumbling times in TIP4P/2005 and TIP3P water differ substantially, tumbling is much slower than internal dynamics of the $\mathrm{N}-\mathrm{H}$ vectors for both water models, explaining the similar backbone amide $S_{\mathrm{NH}}{ }^{2}$ order parameters in TIP4P/2005 and TIP3P water extracted from the LS model.

This work aims at a quantitative comparison of side-chain dynamics from MD and NMR, which demands accurate spectral densities from MD and hence correct global tumbling time scales. The NMR relaxation experiments yield a global tumbling time for $\mathrm{T} 4 \mathrm{~L}$ of $\tau_{\text {iso }}=10.70 \mathrm{~ns}$ (see above), which is very close to $\tau_{\text {iso }}=10.78$ ns obtained from the MD simulations in TIP4P/2005 water. $\ddagger$ Therefore, in the following the data from the TIP4P/2005 simulations are used.

\# Including a hydrodynamic correction to account for box-size dependence ${ }^{66}$ yields $9.30 \mathrm{~ns}$.

\subsection{Comparison of methyl order parameters from MD and NMR}

After having established that the overall rotational diffusion is realistically described in the MD simulations with the TIP4P/ 2005 water model, we next turn to the analysis of simulations in terms of the internal dynamics of methyl groups. Several methods have been proposed for calculating order parameters from MD simulations. In addition to the widespread long-time limit approach (eqn (2)), we used the method proposed by Maragakis and coworkers ${ }^{44}$ for calculating backbone $\mathrm{NH}$ order parameters by directly fitting the total TCFs with a Lipari-Szabo model. Here, we applied both these approaches to the $\mathrm{C}-\mathrm{C}_{\text {methyl }}$ vectors. The results are compared to the spectral density mapping approach, which we applied to $\mathrm{C}_{\text {methyl }}-\mathrm{H}_{\text {methyl vectors. As detailed }}$ in Methods, the total TCFs of the $\mathrm{C}_{\text {methyl }}-\mathrm{H}_{\text {methyl }}$ bond vector reorientation motions were factorized into TCFs for internal motions and overall tumbling; the latter is described by an anisotropic diffusion tensor. This tensor is (again) obtained from MD simulations, and our approach does thus not draw on any experimental NMR information§ or system-specific adjustable parameters. The spectral densities from MD, obtained by Fourier transformation of the TCF, were fitted to the LS2 and LS3 models. The MD-based spectral densities and TCFs are shown in Fig. S1 and $\mathrm{S} 3$, ESI, $\dagger$ respectively. We compare the methyl order parameters obtained with all three methods to the values obtained from our NMR relaxation experiments analyzed with the anisotropic (prolate) model.

Fig. 2 shows the correlation between MD and NMR; the correlation coefficients and RMSD values are also listed in Table 2 . The spectral density mapping approach yields the best agreement with the NMR relaxation experiments, with an RMSD of 0.11 and correlation coefficients of about 0.75 (Fig. 2C). We attribute this result to the fact that the MD-based spectral density mapping approach is very similar in nature to the way the experimental NMR relaxation data is analyzed, and, most importantly, that it properly takes the anisotropic protein diffusion tensor into account. Direct fitting of the raw TCF to a LS model yields comparably accurate results as spectral density mapping (Fig. 2B and Table 2), as this approach also accounts for the decay of the TCF due to anisotropic tumbling. However, the raw TCFs from the MD simulations suffer from statistical noise at long lag-times, which is avoided in the spectral density approach due to the smoothening of both $C_{\text {int }}(t)$ and $C_{\mathrm{O}}(t)$ by the 6- and single-exponential fits, respectively (see Methods; the validity of these assumed functional forms was previously demonstrated for ubiquitin ${ }^{61}$ ). Interestingly, Fig. 2B shows that the direct TCF fits tend to yield too high methyl order parameters. To further investigate this effect, an additional analysis was carried out in which we discriminated between methyl groups described by the LS2 and LS3 models (as determined from the NMR experiments). To that end, for the LS2 methyls, $\tau_{\mathrm{R}}$ was not used as a fitting parameter but set to the fixed value obtained from the MD-based diffusion tensor

$\S$ Except from the choice of the LS2 or LS3 model, see below. 


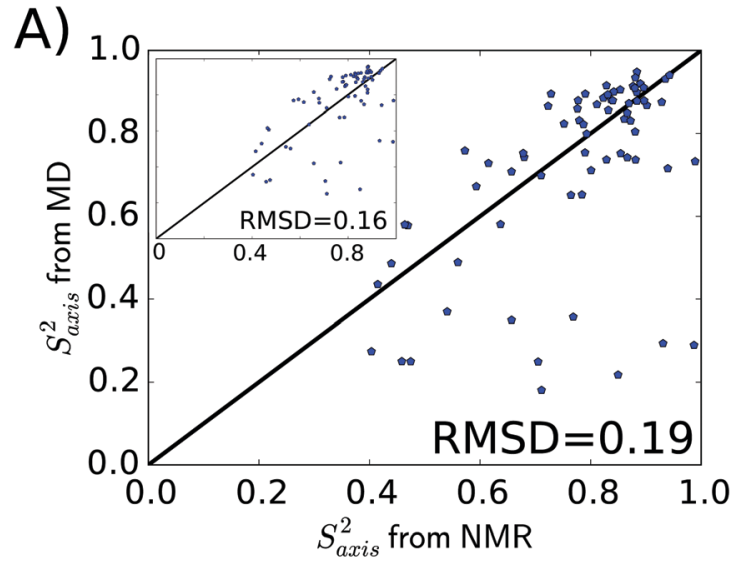

B)

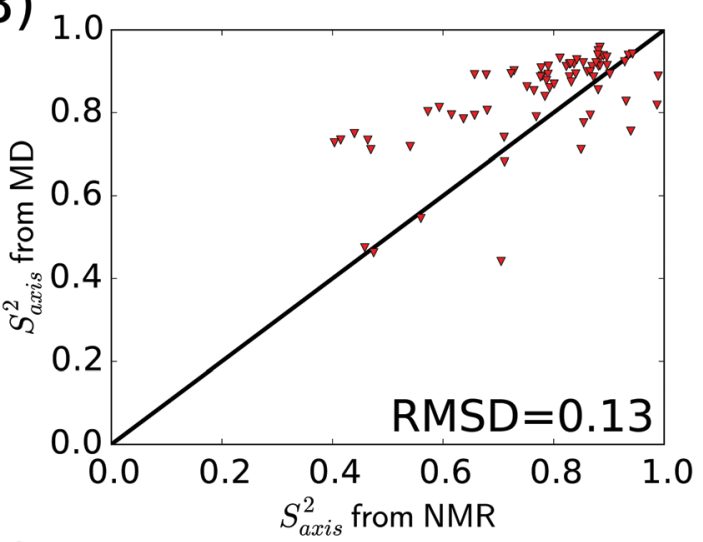

C)

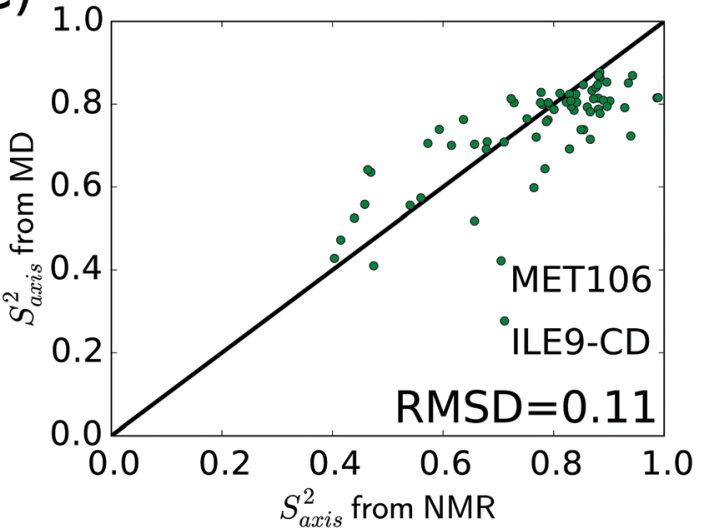

Fig. 2 Correlation of methyl order parameters of T4 lysozyme from NMR relaxation and MD simulations using (A) the long-time limit approach, (B) direct fitting of the Lipari-Szabo TCF, and (C) spectral density mapping. The inset in $(A)$ shows the order parameters obtained from the value of $C_{\text {int }}(t)$ around $\tau_{R}$ instead of in the long-time limit; these $S_{\text {axis }}{ }^{2}$ were obtained by averaging over $C_{\text {int }}(t)$ for lag-times between 9 and $11 \mathrm{~ns}$.

(see Methods). In principle, this approach is the real time space correspondence of the spectral density mapping in Fourier time space. However, as shown in Fig. S4, ESI, $\dagger$ the results are very similar to those shown in Fig. 2B. The RMSD in $S_{\text {axis }}{ }^{2}$ is $0.12, R_{\mathrm{P}}=0.70$, $R_{\mathrm{S}}=0.67$, and the MD order parameters are on average still higher than the NMR ones. This result demonstrates the robustness of the approach. A possible explanation why the direct TCF fitting approach tends to overestimate $S_{\text {axis }}{ }^{2}$ is provided in Section 2.5 below.
Table 2 Pearson $\left(R_{\mathrm{P}}\right)$ and Spearman $\left(R_{\mathrm{S}}\right)$ correlation coefficients and RMSD between MD and NMR for $S_{\text {axis }}{ }^{2}$. The long-time limit, Lipari-Szabo TCF fitting, and spectral density mapping approaches for analysing the MD simulations are compared

\begin{tabular}{llll}
\hline Method & $R_{\mathrm{P}}$ & $R_{\mathrm{S}}$ & RMSD \\
\hline Long-time limit & 0.56 & 0.59 & 0.19 \\
Lipari-Szabo TCF fit & 0.65 & 0.65 & 0.13 \\
Spectral density mapping & 0.74 & 0.76 & 0.11
\end{tabular}

Compared to the previous two methods that take anisotropy into account, taking the long-time limit of $C_{\text {int }}$ yields considerably worse results (Fig. $2 \mathrm{~A}$ and Table 2). There are several methyl groups with high NMR order parameter $\left(S_{\text {axis }}^{2}>0.7\right)$ that have a too low MD order parameter with the long-time limit method. These methyl groups undergo motions on slow time scales $\tau \geq \tau_{\mathrm{R}}$, which contribute to $C_{\text {int }}$ in the long-time limit but are not detectable by NMR spin relaxation, because the TCF has already decayed to zero beyond $\tau_{R}$. This cannot be remedied by simply taking the value of $C_{\text {int }}$ at the overall tumbling time $\tau_{\mathrm{R}}$ instead of in the long-time limit (Fig. 2A, inset). This procedure does only slightly decrease the RMSD between MD and NMR (from 0.19 to 0.16 ) by eliminating some of the too low MD order parameters, as it still neglects the smooth decay of the TCF due to anisotropic protein tumbling.

To judge the above results, the findings reported here for T4L can be compared to previous MD simulation studies of ubiquitin, in which methyl order parameters from MD and NMR were compared. ${ }^{23,25,50,67}$ The reported RMSD in $S_{\text {axis }}{ }^{2}$ between MD and NMR range between $0.104^{23}$ and ca. $0.15 ;^{25}$ some of these studies ${ }^{23,50}$ used the long-time limit approach. These results are comparable to our own ubiquitin simulations, ${ }^{61}$ in which we obtained an RMSD of 0.13 using the spectral density mapping approach to analyze the MD simulations. Hence, for the small isotropic protein ubiquitin, the simple long-time limit and the spectral density mapping approaches seem to yield comparable methyl order parameters. Interestingly, employing the long-time limit approach, Kasinath and coworkers ${ }^{23}$ reported a considerably worse agreement between MD and NMR for larger proteins, such as hen egg white lysozyme, calmodulin, and cytochrome $c 2$. Our present work shows that by taking the decay of the TCF due to anisotropic protein tumbling into account in the analysis of the MD simulations, the same level of agreement with experimental NMR relaxation data can be achieved for a protein as large and complex as $\mathrm{T} 4 \mathrm{~L}$ as was possible before for small isotropic proteins such as ubiquitin.

In addition, to test the quality of the predictions, it is illustrative to compare the MD-derived methyl order parameters for T4L to a "null model" that simply assigns, to each type of methyl group, the average $S_{\text {axis }}{ }^{2}$ determined from NMR relaxation for a range of proteins, ${ }^{21,68}$ i.e., averaging out all siteto-site variations between methyl groups of the same type. To that end, $S_{\text {axis }}{ }^{2}$ values of 12 different proteins were extracted from the literature and compared to our NMR values for T4L (Fig. S5, ESI $\dagger$ ). The null model yields an overall rather poor prediction of the actual order parameters of T4L $\left(\mathrm{RMSD}=0.21, R_{\mathrm{P}}=0.47, R_{\mathrm{S}}=0.51\right)$. 
Given the large computational effort involved in running the MD simulations, it is somewhat disappointing to notice that the longtime limit approach is only slightly better than the "null model". In contrast, the MD-based spectral density mapping and direct TCF fitting methods perform substantially better, and are able to successfully predict the site-to-site variability between methyl groups. This is an encouraging result, because one is often interested in the difference $\Delta S_{\text {axis }}{ }^{2}$ of a (set of) methyl group(s), e.g., upon mutation, binding of a ligand, changes of external conditions, etc. However, at the same time, the agreement between MD and NMR is still worse for side-chain methyl groups than for the backbone (the RMSD in $S_{\text {axis }}{ }^{2}$ is 0.11 , as compared to 0.05 for $S_{\mathrm{NH}}{ }^{2}$, see Fig. 1), showing that there is still need for future improvements.

\subsection{Can Lipari-Szabo order parameters represent methyl group dynamics adequately?}

In the previous section it was observed that the best agreement between NMR and MD was 0.11 (RMSD) for the methyl axis order parameter. Although lower than for most other comparisons to date, the discrepancy between computation and experiment is about twice that observed for backbone dynamics ( $c f$. Fig. 1), and we examine below possible reasons for this discrepancy.

In folded proteins, side-chain dynamics are much richer than those of the backbone, both in time scale and amplitude of the motions: methyl groups are positioned at the ends of side-chains of varying number of bonds relative to the main chain, and may undergo multiple rotamer transitions as well as experience librational motion in each rotamer well along the chain. Thus, the internal TCF decays by the simultaneous action of all these motions, which for the more dynamic sidechains may range from sub-ps to several ns and beyond. To investigate this quantitatively the following analysis is based solely on analyses of the MD trajectories: a comparison was made of order parameters obtained from (i) fitting the internal TCF by a multiple-exponential decay (eqn (16)) and (ii) the internal TCF computed by Lipari-Szabo analysis. In the latter case we first computed the spectral density points from the MD simulation including tumbling, and then fitted $S_{\text {axis }}{ }^{2}, \tau_{\mathrm{f}}$ (LS2) or $S_{\text {axis }}{ }^{2}, \tau_{\mathrm{f}}, \tau_{\mathrm{c}}^{\text {eff }}$ (LS3). $S_{\text {axis }}{ }^{2}$ and $\tau_{\mathrm{f}}$ were used to calculate $C_{\text {int }}(t)$ with eqn (4) in both instances.

Fig. 3 shows example dynamics for two ILE side-chains that display markedly different mobility. Fig. 3A displays data for the $\mathrm{C}^{\delta, 1}$ methyl group of ILE150, which displays three-fold rotation of the methyl group. The ILE150 side-chain is otherwise largely immobile, with dynamics confined to single rotamer wells. Trajectory analysis shows only exceedingly rare excursions to alternative $\chi_{1}$ or $\chi_{2}$ angles during the simulation (Fig. S6 in ESI $\dagger$ ). The internal TCF of the methyl $\mathrm{C}-\mathrm{H}$ vector was fitted by eqn (16), and is shown in red. Fits by the Lipari-Szabo functions LS2 and LS3 are shown in cyan and magenta, respectively, and are virtually superimposable. This good agreement indicates that the simple LS2 model is sufficient to capture the rather limited dynamics of ILE150-C $\mathrm{C}^{\delta, 1}$, and can be adequate for extracting the time constant for methyl spinning (about 5 ps) as well as the order parameter (see inset). In contrast, the $\mathrm{C}^{\delta, 1}$
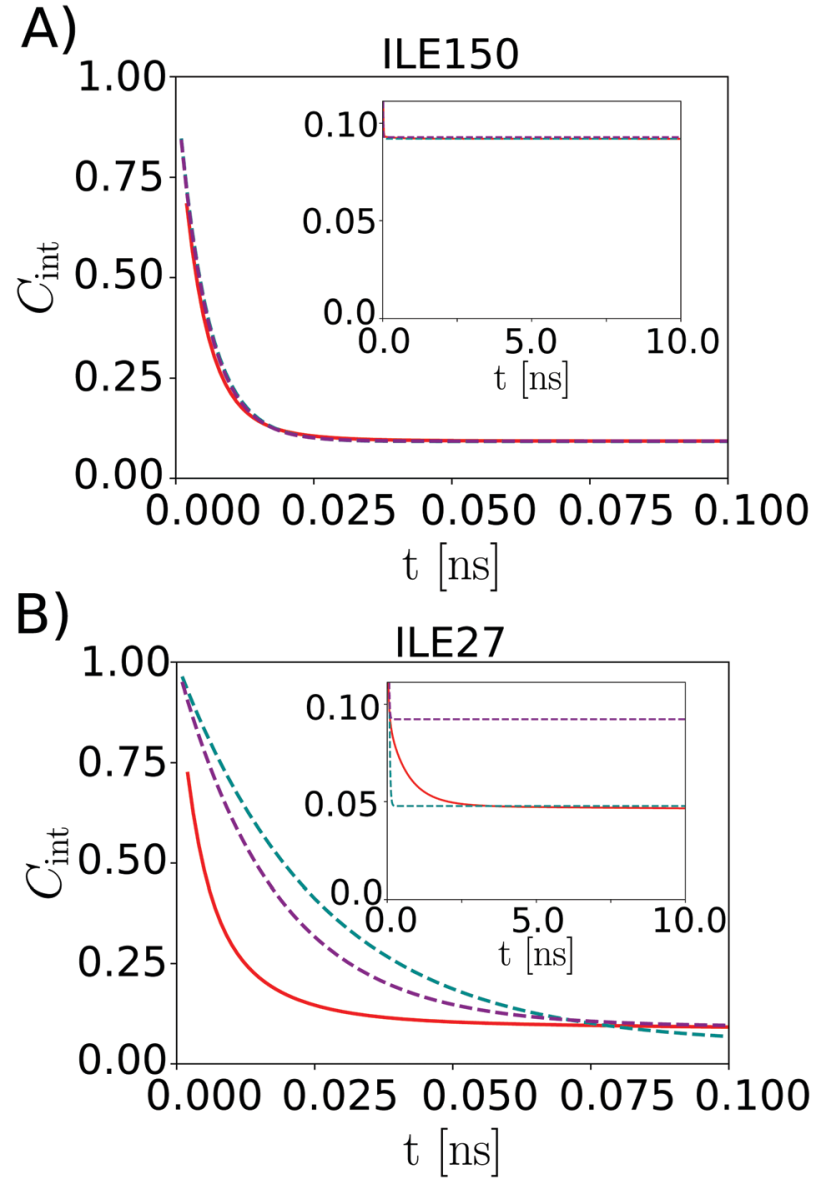

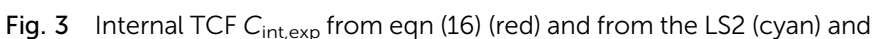
LS3 model (magenta) for ILE150-C $C^{\delta, 1}(\mathrm{~A})$ and ILE27- $\mathrm{C}^{\delta, 1}(\mathrm{~B})$.

methyl group of ILE27 (Fig. 3B) undergoes much more intricate dynamics. As can be seen from the red curve in Fig. 3 and from trajectory analysis (Fig. S6 in ESI $\dagger$ ), the methyl group rotates on a 5 ps time scale about the methyl axis, but it also undergoes frequent two-site jumps about the $\chi_{2}$ angle on a time scale of about 1-2 ns. The presence of this additional dynamic mode causes the internal TCF to decay further over multiple nanoseconds. The result of this complex motion is that neither the LS2 nor the LS3 model fit the internal TCF correctly over the entire range. Surprisingly, LS2 fitting is able to provide a good estimate for $S_{\text {axis }}{ }^{2}$, but at the expense of a large overestimate for the time scale of fast dynamics. LS3 fitting, on the other hand, leads to a strong overestimation of the order parameter, while also still overestimating $\tau_{\mathrm{f}}$. To gauge how well the LS functions fit the internal TCFs for all methyl groups, we compared the agreement of the two curves by computing the following root mean square relative error (RMSRE),

$$
\mathrm{RMSRE}=\frac{1}{N} \sqrt{\sum_{N}\left(\frac{C_{\mathrm{int}, \mathrm{LS}}(t)-C_{\mathrm{int}, \exp }(t)}{C_{\mathrm{int}, \exp }(t)}\right)^{2}},
$$

where $N$ is the number of time points in the TCF, and $C_{\mathrm{int}, \mathrm{LS}}(t)$ and $C_{\text {int,exp }}(t)$ represent the Lipari-Szabo and multi-exponential 
TCFs (eqn (16)), respectively. These RMS relative errors express the relative goodness-of-fit of Lipari-Szabo fitting. The sorted RMSRE for LS2 and LS3 are collected in Fig. S7 in ESI. $\dagger$ Good agreement (RMSRE $<1 \%$ ) is obtained for most TCFs, but a number of methyl groups show poor agreement, as exemplified above for ILE27. In those cases, in NMR LS3 fitting of the spectral density function is statistically better than LS2 fitting, as judged by the Akaike information criterion (AIC), but LS3 fits are still poor and do not approximate the internal TCFs well at all. Fig. 4A shows the agreement obtained for the long-time limit (from eqn (16), i.e., internal TCF $C_{\text {int,exp }}(t)$ without tumbling) and Lipari-Szabo order parameters from the MD data. Order parameters obtained by Lipari-Szabo analysis are systematically overestimated, but LS2 order parameters emerge as better estimates of the internal TCF. Apparently, the improved fitting of the spectral density function with the LS3 equation comes at a cost of systematically worsening the determination of $S_{\text {axis }}^{2}$. Although the present analysis of data for a single protein at a single magnetic field should be considered preliminary, the large discrepancy obtained for LS3 dynamics warrants further investigation.

As a final analysis we considered what happens if LS2 is enforced on fitting the MD data. The result, shown in Fig. 4B,
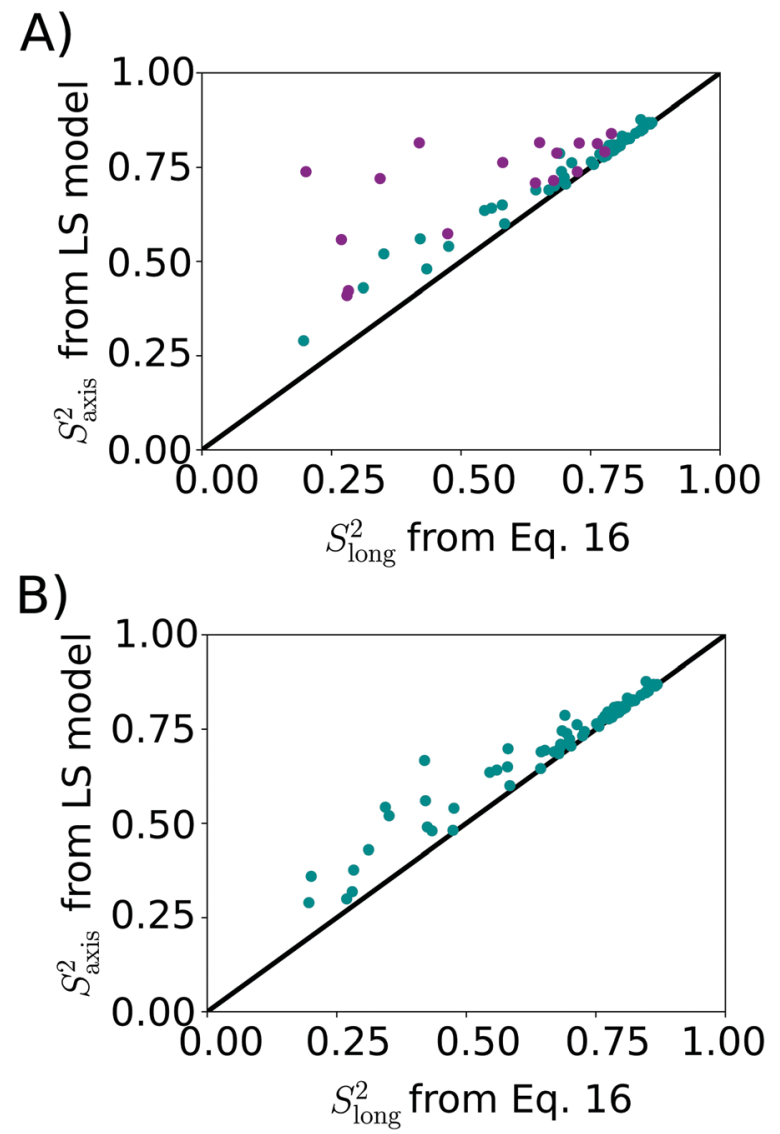

Fig. 4 Correlation between methyl axis order parameter from LS model (LS2: cyan, LS3: magenta) and $\mathrm{S}_{\text {long }}{ }^{2}$ (eqn (16)). (A) LS2/LS3 model picked via AIC. The RMSD between the two data sets is $0.13, R_{\mathrm{P}}=0.77, R_{\mathrm{S}}=0.83$. (B) LS2 model used for all methyl groups. RMSD $=0.06, R_{\mathrm{P}}=0.97, R_{\mathrm{S}}=0.98$. indicates that a rather good agreement is obtained, with an RMSD of 0.06 between the two datasets. Thus, somewhat counterintuitively, LS2 fitting approximates the amount of order surprisingly well, despite yielding poorer fits of the experimental data. This outcome underlines that the estimation of the amount of order from NMR spin relaxation data is challenging, as simple functional forms for the TCF are not able to fully capture the convoluted dynamics of mobile methyl-containing side-chains.

\subsection{Relaxation rates}

As the above section shows, Lipari-Szabo analysis may lead to systematic errors for side-chain dynamic parameters, and hence it is desirable to sidestep this kind of analysis, as the spectral density function can be extracted directly from computation. A first test would be if the MD simulations are able to accurately predict the nuclear spin relaxation rates. This has previously been complicated by unrealistic protein tumbling times in commonly used water force fields, but Section 2.3 indicates that this problem is solved with improved water models and longer simulations.

Fig. 5 compares the relaxation rates $R\left(D_{z}\right), R\left(3 D_{z}{ }^{2}-2\right)$ and $R\left(D_{y}\right)$ from the computed spectral densities to the relaxation rates that were directly measured by NMR relaxation, which does not require any (simplified) motional models. All rates are listed in Table S4, ESI. $\dagger$

In general, the relaxation rates obtained from the MD-based spectral density mapping approach match the experimental relaxation rates. For all three measured relaxation rates, the correlation coefficients between calculated rates from MD and NMR are higher than 0.7 (see Table 3). The relative RMSD of the relaxation rates $R\left(D_{z}\right), R\left(3 D_{z}^{2}-2\right)$, and $R\left(D_{y}\right)$ between MD and NMR are $0.67,0.77$ and 0.17 , respectively. This shows that $R\left(D_{y}\right)$, which contributes most to the spectral density at zero frequency, $J(0)$, and therefore to the methyl order parameter, can be obtained with very high accuracy, whereas the rates $R\left(D_{z}\right)$ and $R\left(3 D_{z}^{2}-2\right)$, which contribute to the spectral densities at higher frequencies, deviate more strongly from experiment. This result might also influence the interpretation of the methyl motions. The slow motions, represented by $J(0)$, contribute to the agreement between MD and NMR in $S_{\text {axis }}{ }^{2}$. Fast motions, e.g., on the time scale of $\tau_{\mathrm{f}}$, determine the spectral density at higher frequencies, which is dominated by $R\left(D_{z}\right)$ and $R\left(3 D_{z}{ }^{2}-2\right)$. Fig. 6 shows that despite the higher relative RMSD in these two rates, the agreement between MD and NMR in $\tau_{\mathrm{f}}$ is decent, too. However, the spread in the $\tau_{\mathrm{f}}$ values in Fig. 6 is larger than that in $S_{\text {axis }}{ }^{2}$ (Fig. 2, bottom panel), suggesting that the MD simulations better recapitulate the amplitude of methyl dynamics than their precise time scales. This conclusion is in line with earlier work. ${ }^{45,56,61}$

Notably, the relaxation rates of one particular methyl group, ALA146- $\mathrm{C}^{\beta}$, are much higher in MD than in NMR (by a factor of 2-3, see Table S4, ESI $\dagger$ ). Similar deviations have been observed previously in MD simulations of staphylococcal nuclease, ${ }^{45}$ which yielded a broader range of methyl rotation correlation times than observed in NMR. Close inspection of our simulations revealed that the rotation of the ALA146 methyl group around its 3-fold symmetry axis is strongly slowed down. The ALA146 methyl group is in a sterically confined local microenvironment, being 
A)

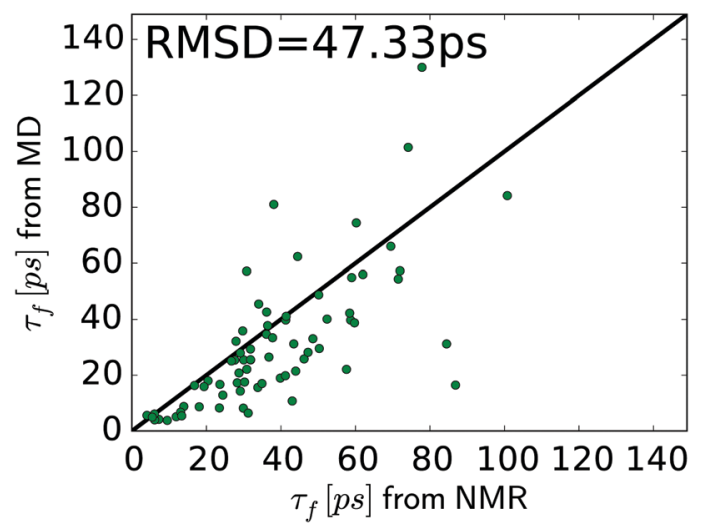

Fig. 6 Correlation of $\tau_{\mathrm{f}}$ for T4L methyl groups from NMR and MD. The RMSD is 47.3 ps, and the Pearson and Spearman correlation coefficients are $R_{\mathrm{P}}=0.67$ and $R_{\mathrm{S}}=0.78$, respectively. The value of $\tau_{\mathrm{f}}$ for ALA146 is not shown (491 ps in MD, 129 ps in NMR).

these $\pi$-systems affect the dynamics of the ALA146 methyl group and to which extent the force field can capture these interactions are intriguing questions but beyond the scope of this work.

\section{Conclusions}

The present work combines NMR relaxation experiments and MD simulations of T4 lysozyme to investigate the side-chain dynamics, as encoded in the deuterium relaxation of methyl groups. It is shown that to obtain accurate results, it is imperative to take protein anisotropy into account in the analysis of both the experimental NMR relaxation data and the MD simulations. Near-quantitative agreement between MD and NMR for generalized order parameters of methyl groups $S_{\text {axis }}{ }^{2}$ and associated correlation times $\tau_{\mathrm{f}}$ was achieved by properly accounting for the decay of the time correlation functions due to anisotropic protein tumbling in solution, which is adequately captured by the TIP4P/ 2005 water model. The employed MD-based spectral density mapping approach closely mimics the way the experimental NMR data is analyzed; directly fitting the raw TCFs, as obtained from the MD simulations, to an extended Lipari-Szabo model yielded almost comparably accurate results, with somewhat too high $S_{\text {axis }}{ }^{2}$. Both these approaches do not draw on any experimental NMR information or adjustable parameters that are specific to a particular system and hence enable true predictions from the MD simulations, with the only reservation that the statistical noise in the MD simulations render it challenging to pick the same LipariSzabo model (LS2 or LS3) as in NMR. In addition to $S_{\text {axis }}{ }^{2}$ and $\tau_{\mathrm{f}}$, which are derived from the data using Lipari-Szabo motional models, good agreement between experiments and simulations is also seen for the spectral densities and relaxation rates that are directly accessible to NMR deuterium relaxation without the need to invoke simplified motional models, which might be problematic for complex side-chain dynamics.

Furthermore, we describe and critically discuss the LEU strong coupling effect, which can compromise deuterium NMR relaxation data for uniformly ${ }^{13} \mathrm{C}$-enriched protein samples if the $\mathrm{C}^{\delta}$ and $\mathrm{C}^{\gamma}$ chemical shifts are close to each other. Here, MD simulations 
helped to identify experimental artifacts for many LEU residues in T4 lysozyme, e.g., spurious large differences between the $S_{\text {axis }}{ }^{2}$ order parameters of LEU prochiral methyl groups. Taking all these aspects into account results in overall very good agreement between MD simulations and NMR relaxation.

This work shows that MD simulations can now provide equally accurate predictions for the side-chain dynamics of rather large and anisotropic proteins such as T4 lysozyme as was previously possible for small isotropic proteins such as ubiquitin. This opens new ways for scrutinizing protein force fields against NMR relaxation data, fueling ongoing efforts to improve the accuracy of the potential energy functions used in the simulations. The improvements described in this study will aid MD simulations in guiding the interpretation of NMR relaxation experiments, as shown here for side-chain methyl dynamics. These developments could also be helpful for deriving improved analytical models that are able to more accurately capture the complex dynamics of side-chains than the highly simplified motional models that have been originally derived for backbone dynamics but are widely used today also for side-chains. Finally, given that close links have been established between NMR order parameters and conformational entropy, ${ }^{23,69-74}$ improvements in the accuracy of the parameters obtained from MD simulations might enable to revisit this close connection, e.g., to gauge and - if necessary - recalibrate an empirical entropy meter.

\section{Methods}

\subsection{NMR sample preparation}

Uniformly ${ }^{15} \mathrm{~N}$-labeled T4 lysozyme (WT*, C54T/C97A) was produced in M9 media with ${ }^{15} \mathrm{NH}_{4} \mathrm{Cl}$ as the sole nitrogen source. $50 \%{ }^{2} \mathrm{H}$-enriched ${ }^{15} \mathrm{~N} /{ }^{13} \mathrm{C} /{ }^{2} \mathrm{H}$-labeled samples were prepared from M9 media using a $1: 1$ volume ratio of $\mathrm{H}_{2} \mathrm{O} / \mathrm{D}_{2} \mathrm{O}$ with ${ }^{15} \mathrm{NH}_{4} \mathrm{Cl}$ and ${ }^{13} \mathrm{C}_{6}$-glucose as sole nitrogen and carbon sources, respectively. Purified protein samples were dialyzed into buffer containing $50 \mathrm{mM}$ sodium phosphate and $25 \mathrm{mM} \mathrm{NaCl}$ at $\mathrm{pH}$ 5.5. NMR samples were prepared in $50 \mathrm{mM}$ phosphate buffer with $25 \mathrm{mM} \mathrm{NaCl}, \mathrm{pH} 5.5,93 \% \mathrm{H}_{2} \mathrm{O} / 7 \% \mathrm{D}_{2} \mathrm{O}(\mathrm{v} / \mathrm{v}){ }^{75}$

\subsection{NMR spectroscopy}

All NMR spectra were collected on Bruker 500, 700, and $950 \mathrm{MHz}$ spectrometers. Temperature was calibrated to $25{ }^{\circ} \mathrm{C}$ using a methanol $\mathrm{d}_{4}$ standard. Backbone ${ }^{15} \mathrm{~N},{ }^{1} \mathrm{H}$ and side-chain methyl ${ }^{13} \mathrm{C},{ }^{1} \mathrm{H}$ resonance assignments of ${ }^{15} \mathrm{~N} /{ }^{13} \mathrm{C} /{ }^{2} \mathrm{H}$-labeled $\mathrm{WT}^{*}$ T4L were obtained primarily through analysis of 3D HNCACB, $\mathrm{CC}(\mathrm{CO}) \mathrm{NH}$, and $\mathrm{H}(\mathrm{CCO}) \mathrm{NH}$. Backbone dynamics were studied by measuring amide ${ }^{15} \mathrm{~N} \mathrm{~T}_{1}, \mathrm{~T}_{2}$, and $\left\{{ }^{1} \mathrm{H}\right\}{ }^{15} \mathrm{~N}$ NOE values at 500 and $700 \mathrm{MHz}$ for $\mathrm{WT}^{*} \mathrm{~T} 4 \mathrm{~L}$ (about $1.0 \mathrm{mM}$ ) through standard experiments. ${ }^{76}$ Side-chain ${ }^{2} \mathrm{H} R\left(D_{z}\right), R\left(D_{y}\right)$ and $R\left(3 D_{z}^{2}-2\right)$ relaxation rates were obtained at $950 \mathrm{MHz}$ for ${ }^{15} \mathrm{~N} /{ }^{13} \mathrm{C} /{ }^{2} \mathrm{H}$-labeled $\mathrm{WT}^{*}$ TL4 (about 1.0 mM), as described previously. ${ }^{16,17}$

\subsection{Analysis of backbone and side-chain dynamics from NMR relaxation}

Backbone and side-chain relaxation rates were obtained by fitting the peak intensity decay as a function of the relaxation delay in the NMR relaxation experiments to a single exponential. The global rotational diffusion parameters were estimated with the programs QUADRIC (www.palmer.hs.columbia.edu) and ROTDIF. ${ }^{77-80}$ The WT* T4L X-ray structure (PDB 1L63) was used in QUADRIC, after centering with PDBINERTIA (www.palmer.hs. columbia.edu). The rigid residues were selected by excluding residues that are subject to fast internal motions and residues with conformational exchange. The former applies if the $\left\{{ }^{1} \mathrm{H}\right\}{ }^{15} \mathrm{~N}$ NOE $<0.65$, and the latter applies if $\left[\left(T_{1, \mathrm{av}}-T_{1, i}\right) / T_{1, \mathrm{av}}+\left(T_{2, \mathrm{av}}-\right.\right.$ $\left.\left.T_{2, i}\right) / T_{2, \text { av }}\right]>$ standard deviation, where $T_{1, \text { av }}$ and $T_{2 \text {,av }}$ are the average values, and $T_{1, i}$ and $T_{2, i}$ are the relaxation times for each individual residue $i$. These conditions identify the rigid residues with relaxation parameter values corresponding to the global rotational dynamics. The local correlation times were obtained through the $R_{2}$ and $R_{1}$ values of the selected rigid residues using R2R1_TM (www.palmer.hs.columbia.edu). The results indicate that $\mathrm{WT}^{*} \mathrm{~T} 4 \mathrm{~L}$ is best approximated by a prolate (axially symmetric) diffusion tensor with a ratio $D_{\|} / D_{\perp}=1.45$. The program DYNAMICS was used to calculate $S_{\mathrm{NH}}{ }^{2}$ from the relaxation data through the model free approach, using a prolate diffusion tensor and a fixed chemical shift anisotropy of $-172 \mathrm{ppm} .{ }^{81,82}$ $S_{\text {axis }}{ }^{2}, \tau_{\mathrm{f}}$, and $\tau_{\mathrm{c}}^{\text {eff }}$ of methyl side-chains were obtained from the relaxation rates of ${ }^{2} \mathrm{H}$ nuclei in $\mathrm{CH}_{2}{ }^{2} \mathrm{H}$ isotopomers by nonlinear least-squares optimization. ${ }^{15}$ Methyl-specific $\tau_{\mathrm{R}, i}$ values were calculated using the orientation of the $\mathrm{C}-\mathrm{C}_{\text {methyl }}$ vector with respect to the diffusion frame from $\tau_{\mathrm{R}, i}=1 / 6\left(D_{\text {iso }}-P_{2}(\cos \theta)\left(D_{\|}-D_{\perp}\right) / 3\right)$ with $\left(D_{\text {iso }}, D_{\|}, D_{\perp}\right)=(1.6,2.0,1.4) \times 10^{7} \mathrm{~s}^{-1}$, and the PDB file produced by QUADRIC where the molecular structure is aligned with the PAF of the diffusion tensor. Deuterium relaxation rates for each methyl group were used as input in model free approaches LS2 and LS3 using a quadrupolar coupling constant of $167 \mathrm{kHz}$. The selection of the LS2 (with fit parameters $S_{\text {axis }}{ }^{2}, \tau_{\mathrm{f}}$ ) or the LS3 model $\left(S_{\text {axis }}{ }^{2}, \tau_{\mathrm{f}}, \tau_{\mathrm{c}}^{\text {eff }}\right)^{15}$ was done by the Akaike information criterion (AIC) test.

\subsection{MD simulations}

All MD simulations were carried out with Gromacs version 5.0.6. ${ }^{83}$ The X-ray structure of the cysteine-free T4L SER44GLY mutant (PDB 107L) ${ }^{84}$ was used as starting structure for our simulations, after changing GLY at position 44 back to SER. Crystallographic water molecules were kept. The protein was centered in a periodic truncated dodecahedron box with a minimum distance between protein and the box boundary of $1.2 \mathrm{~nm}$. The system was solvated with 12221 TIP3P $\mathrm{P}^{57}$ or TIP4P/ $2005^{58}$ water molecules. Sodium and chloride ions were added at a concentration of $0.15 \mathrm{~mol}^{-1}$ to yield an overall neutral simulation system. Prior to MD simulation, the system was energy-minimized (5000 steps steepest descent) and equilibrated in the NPT ensemble for $200 \mathrm{ps}$ with harmonic position restraints on all protein heavy atoms (with force constants of $1000 \mathrm{~kJ} \mathrm{~mol}^{-1} \mathrm{~nm}^{-2}$ ). The Amber ff99SB*-ILDN protein force field ${ }^{27,59,60}$ was used, including our recent adjustments of the methyl rotation barriers. ${ }^{61}$ To keep the temperature constant at $300 \mathrm{~K}$, the velocity rescaling thermostat of Bussi and coworkers ${ }^{85}$ was applied, with coupling time constants of $\tau_{\mathrm{T}}=0.1 \mathrm{ps}$. Isotropic Parrinello-Rahman pressure coupling was used to maintain constant 1 bar pressure, 
with a 2 ps coupling time constant and a compressibility of $4.5 \times$ $10^{-5}$ bar $^{-1}$. The SETTLE ${ }^{86}$ and $\operatorname{LINCS}^{87}$ algorithms were applied to constrain the internal degrees of freedom of water molecules and the bonds in the protein, respectively, allowing for integrating the equations of motion with 2 fs time steps. Lennard-Jones $(6,12)$ interactions were smoothly shifted to zero at a cut-off distance of $1.0 \mathrm{~nm}$; this cut-off distance was also used for the short-range Coulomb interactions. Analytical dispersion corrections were added to energy and pressure to correct for the truncation of the Lennard-Jones interactions. Long-range Coulomb interactions were treated with the particle mesh Ewald (PME) method ${ }^{88}$ with a $0.12 \mathrm{~nm}$ grid spacing and cubic spline interpolation. Finally, ten $300 \mathrm{~ns}$ production MD simulations were carried out in the NPT ensemble using different random seeds for generating initial velocities from a Maxwell-Boltzmann distribution at $300 \mathrm{~K}$. Coordinates were saved to disk every 1 ps.

\subsection{Calculation of order parameters, tumbling times, and relaxation rates from $\mathrm{MD}$}

Time correlation functions for the $\mathrm{C}-\mathrm{C}_{\text {methyl }}$ and for the three $\mathrm{C}_{\text {methyl }}-\mathrm{H}_{\text {methyl }}^{i}(i=1,2,3)$ vector orientations for all methyl groups of all 10 trajectories are calculated up to a maximum lag-time of $150 \mathrm{~ns}$. The TCFs of the three $\mathrm{C}-\mathrm{H}$ vectors of the same methyl group were averaged. The total TCFs were calculated directly from the trajectory, i.e., without removing overall rotation. Internal TCFs were calculated after aligning all trajectory frames to the initial structure. The TCFs of the 10 individual simulations were averaged.

The methyl order parameters according to the long-time limit approach of eqn (2) were calculated as the average value between $75 \mathrm{~ns}$ and $150 \mathrm{~ns}$ of the internal TCF of the $\mathrm{C}-\mathrm{C}_{\text {methyl }}$ bond vector orientation. For the Lipari-Szabo TCF fitting, ${ }^{44}$ the methyl order parameters and rotational tumbling times were determined by directly fitting the total TCFs of the $\mathrm{N}-\mathrm{H}$ and $\mathrm{C}-\mathrm{C}_{\text {methyl }}$ vectors to eqn (10) up to a lag-time of $30 \mathrm{~ns}$. We used QUADRIC with the initial structure of the MD simulation to convert the rotational tumbling from the laboratory frame to the diffusion frame of the protein using a prolate diffusion tensor. The tumbling times of the $\mathrm{C}-\mathrm{C}_{\text {methyl }}$ vectors were converted to the PAF of the diffusion tensor, obtained from the backbone $\mathrm{N}-\mathrm{H}$ vectors, as described by Lee and Palmer. ${ }^{89}$ Based on the initial structure of our MD simulation, we calculated the second order Legendre polynomial $P_{2}(\cos \theta)$ of the cosine of the orientation of each $\mathrm{C}-\mathrm{C}_{\text {methyl vector with }}$ respect to the principal axis of the diffusion tensor. The diffusion tensor obtained from the $\mathrm{N}-\mathrm{H}$ analysis was diagonalized and its principal values $D_{x x}, D_{y y}$, and $D_{z z}$ were used to calculate the rotational diffusion constant in the isotropic model from the trace, $D_{\text {iso }}=\operatorname{Tr} \mathscr{D} / 3$, and the methyl-specific individual diffusion constants $D_{i}=D_{\text {iso }}-P_{2}(\cos \theta)\left(D_{z z}-D_{y y}\right) / 3$. From this, we calculated the methyl-specific tumbling times $\tau_{\mathrm{R}, i}=1 /\left(6 D_{i}\right)$. A prolate model was used for T4L with $D_{z z}=D_{\|}$and $D_{x x}=D_{y y}=D_{\perp}$ as the principal components of the long and short axes of the diffusion tensor, respectively. We obtained $D_{\|} / D_{\perp}=1.36$ using the initial structure of the simulations (i.e., the energy-minimized X-ray structure); calculating this ratio using 3000 snapshots from the MD simulations yielded values in the range [1.2-1.6], with the same average of 1.36. As an additional check, we repeated our analyses using a snapshot after 300 ns of MD simulation (instead of the initial structure, i.e., the energy-minimized X-ray structure) for projecting the $\mathrm{C}-\mathrm{C}_{\text {methyl }}$ vectors onto the PAF of the diffusion tensor. In this snapshot, $c a$. 15 methyl groups adopted a different rotamer state than in the X-ray structure. However, this did not affect the methyl order parameters, with only one single methyl group showing differences larger than $0.015\left(S_{\text {axis }}{ }^{2}\right.$ of LEU66-C $\mathrm{C}^{\delta, 2}$ changes from 1.04 to 0.98 ).

In the spectral density approach, the internal TCFs of the $\mathrm{C}_{\text {methyl }}-\mathrm{H}_{\text {methyl }}$ vectors (i.e., after removing overall tumbling) were fitted with six exponentials plus an offset, ${ }^{90}$

$$
C_{\text {int, } \exp }(t)=\sum_{i=1}^{6} A_{i} \mathrm{e}^{-t / \tau_{i}}+S_{\text {long }}^{2}
$$

where the plateau value $S_{\text {long }}^{2}$ would correspond to the longtime limit order parameter. Different kinds of motions could be represented by the different $\tau_{i}$ values, although they cannot necessarily be ascribed to a physical meaning. In the fit to eqn (16), $\sum_{i=1}^{6} A_{i}+S_{\text {long }}{ }^{2}=1,0 \leq A_{i} \leq 1,0 \leq S_{\text {long }}^{2} \leq 1$, $0 \leq \tau_{1} \leq 10 \mathrm{ps}, 0 \leq \tau_{2} \leq 50 \mathrm{ps}, 0 \leq \tau_{3} \leq 200 \mathrm{ps}, 0 \leq \tau_{4} \leq$ $500 \mathrm{ps}, 0 \leq \tau_{5} \leq 1000 \mathrm{ps}$ and $\tau_{6} \geq 0$.

The resulting internal TCF was multiplied by a singleexponential ${ }^{15}$ using the anisotropic tumbling time $\tau_{\mathrm{R}, i}$ of the corresponding methyl group

$$
C(t)=C_{\text {int }, \exp }(t) \mathrm{e}^{-t / \tau_{\mathrm{R}, i}}
$$

The methyl group-specific tumbling times were extracted based on the diffusion tensor calculated from the rotational tumbling times of the backbone $\mathrm{N}-\mathrm{H}$ vectors, as obtained by the Lipari-Szabo TCF fitting approach, and the orientation of the $\mathrm{C}-\mathrm{C}_{\text {methyl }}$ vector with respect to the PAF of this diffusion tensor, as described above. These $\tau_{\mathrm{R}, i}$ values were also used for the direct TCF fits with the LS2 model (eqn (5)). The total TCF was converted into a spectral density

$$
J(\omega)=\sum_{i=1}^{6} \frac{A_{i} \tau_{i}^{\text {eff }}}{1+\left(\omega \tau_{i}^{\text {eff }}\right)^{2}}+\frac{S_{\text {long }}^{2} \tau_{\mathrm{R}, i}}{1+\left(\omega \tau_{\mathrm{R}, i}\right)^{2}}
$$

with $\tau_{i}^{\text {eff }}=\left(\tau_{i} \tau_{\mathrm{R}, i}\right) /\left(\tau_{i}+\tau_{\mathrm{R}, i}\right)$. Then, the values of $J(0), J\left(\omega_{\mathrm{D}}\right)$ and $J\left(2 \omega_{\mathrm{D}}\right)$ were fitted to eqn (6) or eqn (11) (with $S^{2}=1 / 9 S_{\text {axis }}{ }^{2}$ ) according to the following procedure:

(1) LS2 grid search within $S_{\text {axis;LS2 }}^{2} \in\left[0,0.01, \ldots, 2 S_{\text {long }}^{2}\right]$ and $\tau_{\mathrm{f} ; \mathrm{LS} 2} \in\left[0 \mathrm{ps}, 1 \mathrm{ps}, \ldots, 2 \tau_{\mathrm{fit}}^{\mathrm{eff}}\right] . S_{\text {long }}{ }^{2}$ and $\tau_{\mathrm{fit}}^{\mathrm{eff}}=\sum_{i=1}^{6} A_{i} \tau_{i}$ were used as initial values for the fitting parameters.

(2) The results from the grid search were used as starting points for a direct, i.e., gradient-based fit of $S_{\text {axis;LS2 }}{ }^{2}$ and $\tau_{\mathrm{f} ; \mathrm{LS} 2}$ to yield the final LS2 parameter pair $S_{\text {axis;LS2 }}{ }^{2} ; \tau_{\mathrm{f} ; \mathrm{LS} 2}$.

(3) LS3 grid search within $S_{\text {axis;LS3 }}{ }^{2} \in\left[0,0.01, \ldots, 2 S_{\text {axis;LS2 }}{ }^{2}\right]$, $\tau_{\mathrm{f} ; \mathrm{LS} 3} \in\left[\tau_{\mathrm{f} ; \mathrm{LS} 2}-50 \mathrm{ps}, \tau_{\mathrm{f} ; \mathrm{LS} 2}-49 \mathrm{ps}, \ldots, \tau_{\mathrm{f} ; \mathrm{LS} 2}+50 \mathrm{ps}\right]$ and $\tau_{\mathrm{c}}^{\text {eff }} \in$ $\left[\tau_{\mathrm{R}, i}-5 \mathrm{~ns}, \tau_{\mathrm{R}, i}-4.9 \mathrm{~ns}, \ldots, \tau_{\mathrm{R}, i}+5 \mathrm{~ns}\right]$. The LS2 results $\left(S_{\text {axis;LS2 }}{ }^{2} ; \tau_{\mathrm{f} ; \mathrm{LS} 2}\right)$ were used to initiate $S_{\mathrm{f} ; \mathrm{LS} 3}{ }^{2}$ and $\tau_{\mathrm{f} ; \mathrm{LS} 3}$. 
Furthermore, the methyl-specific $\tau_{\mathrm{R}, i}$ 's from eqn (17) were used as starting points for fitting $\tau_{\mathrm{c}}^{\text {eff }}$.

(4) Direct fit of $S_{\text {axis;LS3 }}{ }^{2}, \tau_{\mathrm{f} ; \mathrm{LS} 3}$, and $\tau_{\mathrm{c}}^{\text {eff }}$ using the results from the previous grid search as starting points, yielding the final LS3 parameter triple $\left(S_{\text {axis;LS3 }}{ }^{2} ; \tau_{\mathrm{f} ; \mathrm{LS} 3} ; \tau_{\mathrm{c}}^{\text {eff }}\right)$.

At every step of the grid search or gradient-based minimization, $J_{\text {model }}(0), J_{\text {model }}\left(\omega_{\mathrm{D}}\right)$, and $J_{\text {model }}\left(2 \omega_{\mathrm{D}}\right)$ were determined from eqn $(6)$ or eqn (11), and $R_{\text {model }}\left(D_{z}\right), R_{\text {model }}\left(3 D_{z}^{2}-2\right), R_{\text {model }}\left(D_{y}\right)$, and $\chi^{2}$ were calculated from

$$
\chi^{2}=\sum_{i \in\left\{D_{z}, 3 D_{z}^{2}-2, D_{y}\right\}}\left(\frac{R_{\text {model }}(i)-R_{\text {analytical }}(i)}{R_{\text {error }}(i)}\right)^{2}
$$

Minimizing $\chi^{2}$ yielded the fitting parameter pair $\left(S_{\text {axis;LS2 }}{ }^{2} ; \tau_{\mathrm{f} ; \mathrm{LS} 2}\right)$ for eqn (6) or triple $\left(S_{\mathrm{axis} ; \mathrm{LS} 3}{ }^{2} ; \tau_{\mathrm{f} ; \mathrm{LS} 3} ; \tau_{\mathrm{c}}^{\text {eff }}\right)$ for eqn (11) (with $S^{2}=$ $\left.1 / 9 S_{\text {axis }}{ }^{2}\right) . R_{\text {error }}(i)$ are the standard errors of the mean of the 10 relaxation rates $R_{\text {model }}(l)$ from the 10 individual MD simulations; $R_{\text {analytical }}(l)$ are directly derived from $J(0), J\left(\omega_{\mathrm{D}}\right)$, and $J\left(2 \omega_{\mathrm{D}}\right)$ via eqn (18). Finally, for every methyl group, we chose the same model (LS2 or LS3) as was picked for the NMR relaxation data (using AIC, see above), as it is difficult to obtain reliable estimates for the statistical errors of the relaxation rates from the MD simulations.

\section{Conflicts of interest}

There are no conflicts of interest to declare.

\section{Acknowledgements}

We gratefully acknowledge insightful discussions with Kresten Lindorff-Larsen (University of Copenhagen) and Nikolai Skrynnikov (Purdue University). This work was performed using the NMR spectrometers at the Danish Center for Ultra-High Field NMR Spectroscopy. The Deutsche Forschungsgemeinschaft (DFG) supported this work through Cluster of Excellence RESOLV (EXC 1069) and Emmy-Noether grant SCHA 1574/3-1 to L. S. The Steinbuch Centre for Computing (SCC), Karlsruhe/Germany, is acknowledged for providing computational resources.

\section{References}

1 K. Henzler-Wildman and D. Kern, Nature, 2007, 450, 964-972.

2 H. Frauenfelder, S. Sligar and P. Wolynes, Science, 1991, 254, 1598-1603.

3 J. W. Peng, J. Phys. Chem. Lett., 2012, 3, 1039-1051.

4 P. J. Sapienza and A. L. Lee, Curr. Opin. Pharmacol., 2010, 10, 723-730.

5 A. Mittermaier and L. E. Kay, Science, 2006, 312, 224-228.

6 A. G. Palmer, Chem. Rev., 2004, 104, 3623-3640.

7 R. Brüschweiler, Curr. Opin. Struct. Biol., 2003, 13, 175-183.

8 D. A. Case, Acc. Chem. Res., 2002, 35, 325-331.

9 K. Lindorff-Larsen, R. B. Best, M. A. DePristo, C. M. Dobson and M. Vendruscolo, Nature, 2005, 433, 128-132.

10 N. Salvi, A. Abyzov and M. Blackledge, J. Phys. Chem. Lett., 2016, 7, 2483-2489.
11 G. Lipari and A. Szabo, J. Am. Chem. Soc., 1982, 104, 4546-4559.

12 G. Lipari and A. Szabo, J. Am. Chem. Soc., 1982, 104, 4559-4570.

13 B. Halle and H. Wennerström, J. Chem. Phys., 1981, 75, 1928-1943.

14 G. M. Clore, A. Szabo, A. Bax, L. E. Kay, P. C. Driscoll and A. M. Gronenborn, J. Am. Chem. Soc., 1990, 112, 4989-4991.

15 N. R. Skrynnikov, O. Millet and L. E. Kay, J. Am. Chem. Soc., 2002, 124, 6449-6460.

16 D. R. Muhandiram, T. Yamazaki, B. D. Sykes and L. E. Kay, J. Am. Chem. Soc., 1995, 117, 11536-11544.

17 O. Millet, D. R. Muhandiram, N. R. Skrynnikov and L. E. Kay, J. Am. Chem. Soc., 2002, 124, 6439-6448.

18 D. C. Chatfield and S. E. Wong, J. Phys. Chem. B, 2000, 104, 11342-11348.

19 D. Yang, A. Mittermaier, Y. K. Mok and L. E. Kay, J. Mol. Biol., 1998, 276, 939-954.

20 J. J. Chou, D. A. Case and A. Bax, J. Am. Chem. Soc., 2003, 125, 8959-8966.

21 R. B. Best, J. Clarke and M. Karplus, J. Am. Chem. Soc., 2004, 126, 7734-7735.

$22 \mathrm{H}$. Hu, J. Hermans and A. L. Lee, J. Biomol. NMR, 2005, 32, 151-162.

23 V. Kasinath, K. A. Sharp and A. J. Wand, J. Am. Chem. Soc., 2013, 135, 15092-15100.

24 N. Foloppe and A. D. MacKerell, J. Comput. Chem., 2000, 21, 86-104.

25 G. R. Bowman, J. Comput. Chem., 2016, 37, 558-566.

26 Y. Duan, C. Wu, S. Chowdhury, M. C. Lee, G. Xiong, W. Zhang, R. Yang, P. Cieplak, R. Luo, T. Lee, J. Caldwell, J. Wang and P. Kollman, J. Comput. Chem., 2003, 24, 1999-2012. 27 K. Lindorff-Larsen, S. Piana, K. Palmo, P. Maragakis, J. L. Klepeis, R. O. Dror and D. E. Shaw, Proteins, 2010, 78, 1950-1958.

28 J. J. Prompers and R. Brüschweiler, J. Am. Chem. Soc., 2002, 124, 4522-4534.

29 S. Genheden, J. Mol. Graphics, 2017, 71, 80-87.

30 D. E. Woessner, J. Chem. Phys., 1962, 37, 647-654.

31 H. Shimizu, J. Chem. Phys., 1962, 37, 765-778.

32 P. Luginbühl, K. V. Pervushin, H. Iwai and K. Wüthrich, Biochemistry, 1997, 36, 7305-7312.

33 V. Wong and D. A. Case, J. Phys. Chem. B, 2008, 112, 6013-6024.

34 J. S. Anderson, G. Hernández and D. M. LeMaster, J. Chem. Theory Comput., 2017, 13, 3276-3289.

35 P.-c. Chen, M. Hologne and O. Walker, J. Phys. Chem. B, 2017, 121, 1812-1823.

36 M. Linke, J. Köfinger and G. Hummer, J. Phys. Chem. B, 2018, 122, 5630-5639.

37 O. H. S. Ollila, H. A. Heikkinen and H. Iwai, J. Phys. Chem. B, 2018, 122, 6559-6569.

38 Y. E. Ryabov, C. Geraghty, A. Varshney and D. Fushman, J. Am. Chem. Soc., 2006, 128, 15432-15444.

39 R. Brüschweiler, X. Liao and P. E. Wright, Science, 1995, 268, 886-889.

40 G. Barbato, M. Ikura, L. E. Kay, R. W. Pastor and A. Bax, Biochemistry, 1992, 31, 5269-5278.

41 J. M. Schurr, H. P. Babcock and B. S. Fujimoto, J. Magn. Reson., 1994, 105, 211-224. 
42 O. Millet, A. Mittermaier, D. Baker and L. E. Kay, J. Mol. Biol., 2003, 329, 551-563.

43 R. Ishima, A. P. Petkova, J. M. Louis and D. A. Torchia, J. Am. Chem. Soc., 2001, 123, 6164-6171.

44 P. Maragakis, K. Lindorff-Larsen, M. P. Eastwood, R. O. Dror, J. L. Klepeis, I. T. Arkin, M. Ø. Jensen, H. Xu, N. Trbovic, R. A. Friesner, A. G. Palmer and D. E. Shaw, J. Phys. Chem. B, 2008, 112, 6155-6158.

45 D. C. Chatfield, A. Szabo and B. R. Brooks, J. Am. Chem. Soc., 1998, 120, 5301-5311.

46 R. B. Best, J. Clarke and M. Karplus, J. Mol. Biol., 2005, 349, 185-203.

47 J. Huang and A. D. MacKerell, J. Comput. Chem., 2013, 34, 2135-2145.

48 T. Rajitha Rajeshwar, J. C. Smith and M. Krishnan, J. Am. Chem. Soc., 2014, 136, 8590-8605.

49 K. A. Sharp, E. OBrien, V. Kasinath and A. J. Wand, Proteins, 2015, 83, 922-930.

50 E. S. OBrien, A. J. Wand and K. A. Sharp, Protein Sci., 2016, 25, 1156-1160.

51 F. A. A. Mulder, B. Hon, A. Mittermaier, F. W. Dahlquist and L. E. Kay, J. Am. Chem. Soc., 2002, 124, 1443-1451.

52 R. L. Dunbrack and F. E. Cohen, Protein Sci., 1997, 6, 1661-1681. 53 F. A. A. Mulder, ChemBioChem, 2009, 10, 1477-1479.

54 V. Agarwal, A. Diehl, N. R. Skrynnikov and B. Reif, J. Am. Chem. Soc., 2006, 128, 12620-12621.

55 A. L. Lee, J. L. Urbauer and J. A. Wand, J. Biomol. NMR, 1997, 9, 437-440.

56 Y. Xue, M. S. Pavlova, Y. E. Ryabov, B. Reif and N. R. Skrynnikov, J. Am. Chem. Soc., 2007, 129, 6827-6838.

57 W. L. Jorgensen, J. Chandrasekhar, J. D. Madura, R. W. Impey and M. L. Klein, J. Chem. Phys., 1983, 79, 926-935.

58 J. L. F. Abascal and C. Vega, J. Chem. Phys., 2005, 123, 234505.

59 V. Hornak, R. Abel, A. Okur, B. Strockbine, A. Roitberg and C. Simmerling, Proteins, 2006, 65, 712-725.

60 R. B. Best and G. Hummer, J. Phys. Chem. B, 2009, 113, 9004-9015.

61 F. Hoffmann, F. A. A. Mulder and L. V. Schäfer, J. Phys. Chem. B, 2018, 122, 5038-5048.

62 T. Zeiske, K. A. Stafford, R. A. Friesner and A. G. Palmer, Proteins, 2013, 81, 499-509.

63 J. S. Anderson and D. M. LeMaster, Biophys. Chem., 2012, 168-169, 28-39.

64 I.-C. Yeh and G. Hummer, J. Phys. Chem. B, 2004, 108, 15873-15879.

65 S. Tazi, A. Botan, M. Salanne, V. Marry, P. Turq and B. Rotenberg, J. Phys.: Condens. Matter, 2012, 24, 284117.
66 M. Linke, J. Köfinger and G. Hummer, J. Phys. Chem. Lett., 2018, 9, 2874-2878.

67 D. Long, D.-W. Li, K. F. A. Walter, C. Griesinger and R. Brüschweiler, Biophys. J., 2011, 101, 910-915.

68 A. Mittermaier, L. E. Kay and J. D. Forman-Kay, J. Biomol. NMR, 1999, 13, 181-185.

69 M. Akke, R. Brüschweiler and A. G. Palmer, J. Am. Chem. Soc., 1993, 115, 9832-9833.

70 D. Yang and L. E. Kay, J. Mol. Biol., 1996, 263, 369-382.

71 Z. Li, S. Raychaudhuri and A. J. Wand, Protein Sci., 1996, 5, 2647-2650.

72 D. M. LeMaster, J. Am. Chem. Soc., 1999, 121, 1726-1742.

73 K. K. Frederick, M. S. Marlow, K. G. Valentine and A. J. Wand, Nature, 2007, 448, 325-329.

74 C. M. Petit, J. Zhang, P. J. Sapienza, E. J. Fuentes and A. L. Lee, Proc. Natl. Acad. Sci. U. S. A., 2009, 106, 18249-18254.

75 F. A. A. Mulder, B. Hon, D. R. Muhandiram, F. W. Dahlquist and L. E. Kay, Biochemistry, 2000, 39, 12614-12622.

76 N. A. Farrow, R. Muhandiram, A. U. Singer, S. M. Pascal, C. M. Kay, G. Gish, S. E. Shoelson, T. Pawson, J. D. FormanKay and L. E. Kay, Biochemistry, 1994, 33, 5984-6003.

77 D. Fushman, R. Xu and D. Cowburn, Biochemistry, 1999, 38, 10225-10230.

78 R. Varadan, O. Walker, C. Pickart and D. Fushman, J. Mol. Biol., 2002, 324, 637-647.

79 O. Walker, R. Varadan and D. Fushman, J. Magn. Reson., 2004, 168, 336-345.

80 D. Fushman, R. Varadan, M. Assfalg and O. Walker, Prog. Nucl. Magn. Reson. Spectrosc., 2004, 44, 189-214.

81 D. Fushman, S. Cahill and D. Cowburn, J. Mol. Biol., 1997, 266, 173-194.

82 J. B. Hall and D. Fushman, J. Biomol. NMR, 2003, 27, 261-275.

83 M. J. Abraham, T. Murtola, R. Schulz, S. Páll, J. C. Smith, B. Hess and E. Lindahl, SoftwareX, 2015, 1-2, 19-25.

84 M. Blaber, X. J. Zhang and B. W. Matthews, Science, 1993, 260, 1637-1640.

85 G. Bussi, D. Donadio and M. Parrinello, J. Chem. Phys., 2007, 126, 014101.

86 S. Miyamoto and P. A. Kollman, J. Comput. Chem., 1992, 13, 952-962.

87 B. Hess, J. Chem. Theory Comput., 2008, 4, 116-122.

88 U. Essmann, L. Perera, M. L. Berkowitz, T. Darden, H. Lee and L. G. Pedersen, J. Chem. Phys., 1995, 103, 8577-8593.

89 L. K. Lee, M. Rance, W. J. Chazin and A. G. Palmer, J. Biomol. NMR, 1997, 9, 287-298.

90 T. Bremi, R. Brüschweiler and R. R. Ernst, J. Am. Chem. Soc., 1997, 119, 4272-4284. 